\title{
Coupled ocean-atmosphere forecasting at short and medium time scales
}

\author{
by Julie Pullen ${ }^{1,2}$, Richard Allard ${ }^{3}$, Hyodae Seo ${ }^{4}$, Arthur J. Miller ${ }^{5}$, Shuyi Chen ${ }^{6}$, \\ Luciano Ponzi Pezzi ${ }^{7}$, Travis $\mathrm{Smith}^{3}$, Philip Chu ${ }^{8}$, José Alves ${ }^{9}$, and Rui Caldeira9
}

\begin{abstract}
Recent technological advances over the past few decades have enabled the development of fully coupled atmosphere-ocean modeling prediction systems that are used today to support short-term (days to weeks) and medium-term (10-21 days) needs for both the operational and research communities. We overview the coupling framework, including model components and grid resolution considerations, as well as the coupling physics by examining heat fluxes between atmosphere and ocean, momentum transfer, and freshwater fluxes. These modeling systems can be run as fully coupled atmosphere-ocean and atmosphere-ocean-wave configurations. Examples of several modeling systems applied to complex coastal regions including Madeira Island, Adriatic Sea, Coastal California, Gulf of Mexico, Brazil, and the Maritime Continent are presented. In many of these studies, a variety of field campaigns have contributed to a better understanding of the underlying physics associated with the atmosphere-ocean feedbacks. Examples of improvements in predictive skill when run in coupled mode versus standalone are shown. Coupled model challenges such as model initialization, data assimilation, and earth system prediction are discussed.
\end{abstract}

Keywords: Coupled air-sea modeling

\section{Introduction}

Coupled air-sea forecasting systems have been planned and architected over several decades (Hodur 1997; Hodur et al. 2002; Bender and Ginis 2000; Bender et al. 2007). Whereas global coupled models typically have resolutions of approximately $1^{\circ}$, regional

1. Stevens Institute of Technology, Hoboken, New Jersey 07030, USA

2. Corresponding author: e-mail: julie.pullen@stevens.edu

3. Naval Research Laboratory, Stennis Space Center, Mississippi 39529, USA

4. Woods Hole Oceanographic Institution, Woods Hole, Massachusetts 02543, USA

5. Scripps Institution of Oceanography, La Jolla, California 92037, USA

6. University of Washington Seattle, WA 98195

7. National Institute for Space Research, São José dos Campos, São Paulo 12227-010, Brazil

8. NOAA Great Lakes Environmental Research Laboratory, Ann Arbor, Michigan 48108, USA

9. Oceanic Observatory of Madeira, Agência Regional para o Desenvolvimento da Investigacão Tecnologia e Inovacão, Funchal, Portugal, and Instituto Dom Luiz, University of Lisbon, Portugal

(C) 2017 Julie Pullen, Richard Allard, Hyodae Seo, Arthur J. Miller, Shuyi Chen, Luciano Ponzi Pezzi, Travis Smith, Philip Chu, José Alves, and Rui Caldeira. 
coupled models enable high resolution (10 km or less) for local areas. The accumulation of studies employing coupled air-sea models and the opportunity to synthesize their results has encouraged the evolution of coupled modeling systems and their application to many areas around the globe. Coupled systems have been shown to yield benefits across multiple forecast horizons: from short (multiple days) to medium (multiple weeks) timescales. Significant factors and examples in the path of development and application of regional coupled models are described in this contribution.

A growing recognition of the benefits to both realms of air-sea coupled prediction has come with the expanded demonstration by observations that oceanic fronts can modify the overlying atmosphere (Chelton et al. 2004; Pezzi et al. 2004; Small et al. 2008; Chelton and Xie 2010). Coupled models have demonstrated skill in capturing key aspects of coastal frontal dynamics (Perlin et al. 2007; Seo et al. 2008b, 2016) in hurricane prediction (Chen et al. 2007; Seo and Xie 2013) and across numerous regional applications. The positive impacts of coupling can extend through the entire atmospheric boundary layer, including changes to cloud dynamics (Fallmann et al. 2017). In this Introduction we utilize two themes to introduce topics that will be explored in the remainder of this overview paper. These themes are "research to operations" and "multimodel approaches."

The advent of two-way coupled ocean-atmosphere regional modeling has enabled new capabilities in high-resolution prediction. The U.S. Navy's Coupled Ocean/Atmosphere Mesoscale Prediction System (COAMPS ${ }^{\circledR}$ ) has been employed and recognized for its skill in representing complex coastal regions and for its utility in guiding field campaigns (Doyle et al. 2009; Chen et al. 2010; Pullen et al. 2011; Shinoda et al. 2013). Among its strengths are its origin at the interface of oceanography and marine meteorology research groups, its ease in relocating to new domains (Hodur et al. 2002), and its operational deployment throughout the world.

Open-source alternatives exist in the form of coupled Weather Research and Forecasting Model (WRF) and Regional Ocean Modeling System (ROMS) frameworks, such as the Coupled-Ocean-Atmosphere-Wave-Sediment-Transport System (COAWST) (Warner et al. 2010). COAWST has been applied to Adriatic cold air bora events (Ricchi et al. 2016), first studied with two-way coupled COAMPS by Pullen, Doyle, and Signell (2006). We give an update on this Mediterranean domain in the context of "research to operations." Then we probe the results of these two models, COAMPS and COAWST, applied contemporaneously to the same island domain (Section 1b).

\section{a. From research to operations: an evolution}

Accelerated by a series of international field and modeling campaigns over the past decade, coupled air-sea modeling systems are transitioning from research to operations. For example, in early prototype two-way coupled studies for the Adriatic Circulation Experiment (ACE), Pullen, Doyle, and Signell (2006) and Pullen et al. (2007) examined downslope windstorms, or bora, that occur in the topographic mountains of the Dinaric Alps of Croatia 
during late fall and winter months. The circulation patterns in the northern Adriatic Sea are influenced by the bora wind jet as the sea transfers heat to the colder overlying air. In that COAMPS study, the heat flux root-mean-square error (RMSE) was reduced by $30 \%$ on average at four over-water instrumentation sites in the northern Adriatic using two-way coupling. Although the original study utilized coupling at a 6-hour coupling interval, recent operationally-oriented Earth System Modeling Framework (ESMF)-based simulations with a coupling interval on the order of minutes (Allard et al. 2010) for that same region, time period, and locations found a total heat flux RMSE reduction of $44 \%$ when comparing the coupled versus uncoupled runs.

In both studies, the coupled model clearly outperforms the uncoupled model, a result that helped motivate the operational deployment of coupled systems. The two studies further suggest that the coupling frequency influences the dynamics of coupled model simulationsa topic that we will revisit further. But first we probe coupled modeling approaches for a common island setting.

\section{b. A multimodel approach: islands as a coupled model challenge}

Islands represent a challenging and important setting for coupled modeling systems (Caldeira et al. 2016). Ocean features initiated by islands (such as mesoscale eddies, filaments, and warm wakes) generate distinct sea surface temperature (SST) signatures (Sangrà et al. 2007). The strong near-surface wind and ocean surface heat flux gradients occurring in the flanks and lee of mountain terrain are loci of vigorous air-sea interaction (Grubišić, Sachsperger, and Caldeira 2015). The dynamics create an island-modulated circulation that is, fundamentally, a two-way coupled environment, and is therefore a key setting for realistic coupled models to emulate.

Of particular interest in this regard are local impacts generated by submesoscale and mesoscale SST variations that can alter heat fluxes and winds. Warm SSTs are commonly found in the lee of islands (Caldeira et al. 2002). Caldeira and Tomé (2013) speculated that lee-side ocean warming results from the enhanced solar heating of cloud-free atmospheric wakes. Caldeira et al. (2002) and Caldeira (pers. comm.) measured this effect on the island of Madeira to be 2 to $3^{\circ} \mathrm{C}$ through in the upper 10 to $20 \mathrm{~m}$. During summertime, the Madeira warm wake extends for over $100 \mathrm{~km}$. Similar characteristics of warm wakes have been measured in the lee of the Canary Islands (Barton et al. 2000). And wake regions can have even longer-ranging and more-pronounced effects (>300 km) (Smith and Grubišic 1993; Hafner and Xie 2003).

A recent deployment of multiple coupled models, at high resolution, to examine a common island domain yields insight on model performance in this challenging geographical setting. The COAMPS model was configured for the island of Madeira (Figure 1) using a triply-nested domain from 18/6/2-km resolution (Pullen et al. 2017). The ocean model is activated on one domain at $2-\mathrm{km}$ resolution under the third (innermost) atmosphere 


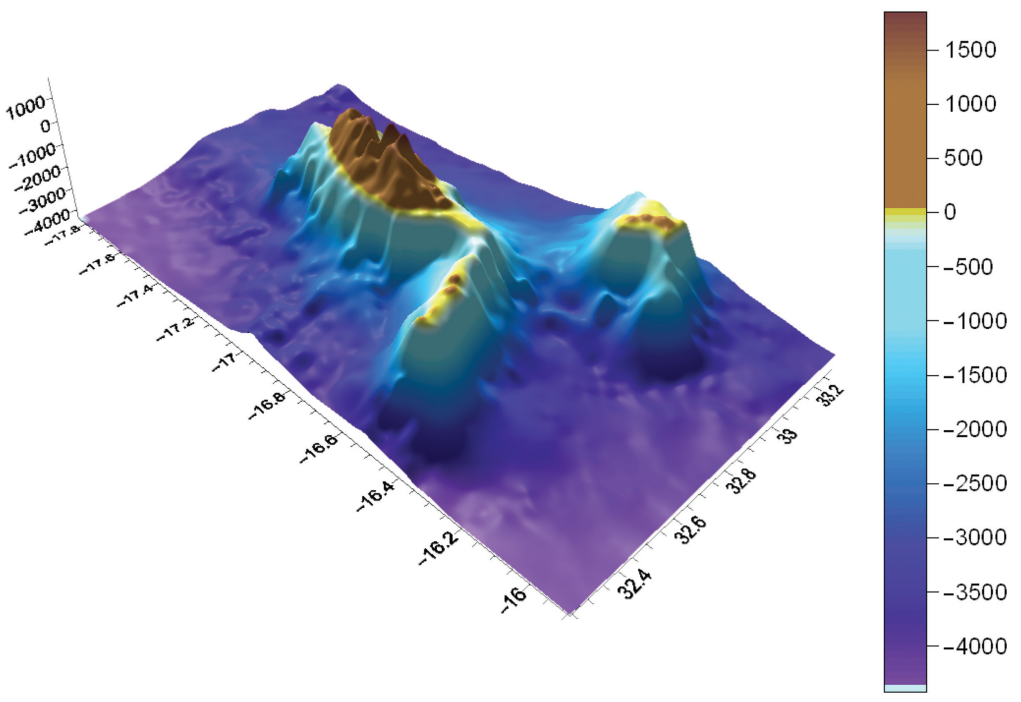

Figure 1. Terrain and bathymetry $(\mathrm{m})$ of the Madeira archipelago in the eastern Atlantic, consisting of the main island of Madeira and the flat islands of Porto Santo to the northeast, and Desertas to the southeast.

model domain. The ocean and atmosphere have 50 and 60 vertical levels, respectively. The atmosphere and ocean models exchange flux information at a time interval of 6 minutes using the ESMF. The COAWST system was applied to Madeira doubly nested with resolutions of 3 and $1 \mathrm{~km}$ (see Appendix $b$ for more details). There are 40 vertical levels in WRF and 32 in ROMS. The variables are exchanged every 5 minutes. The coupling is performed using the Model Coupling Toolkit (MCT) (Larson, Jacob, and Ong 2005; Jacob, Larson, and Ong 2005). The runs were performed for the month of June 2011.

In the simulations both models produce a diurnal warm wake south of Madeira having a magnitude of approximately $2-3^{\circ} \mathrm{C}$ above surrounding waters (Figure 2). However the warming in COAWST is more pronounced and shows stronger warming windward of the island (the "antiwake") compared with COAMPS. Daily satellite SST composites suggest the pattern and amplitude $\left(1-2^{\circ} \mathrm{C}\right)$ of warming but do not permit an assessment of the temporal evolution of the feature. Both models show strong flank "tip jet" winds and a weak wind region in the immediate lee of Madeira (Figure 3). Another distinction is that COAWST produces overall stronger winds whereas COAMPS exhibits terrain-modulated flow from drainage winds (06UTC on 15 June).

The variation between the models is indicative of differences in model configurations and parameterizations. Section 2 describes the general modeling frameworks and parameterization options, and Section 3 details the underlying physics of the coupling choices. Then in Section 4 we examine several case studies of short-term ( $\sim$ days to week) coupled prediction that highlight modeling configuration choices such as the effects of wave 
(a)
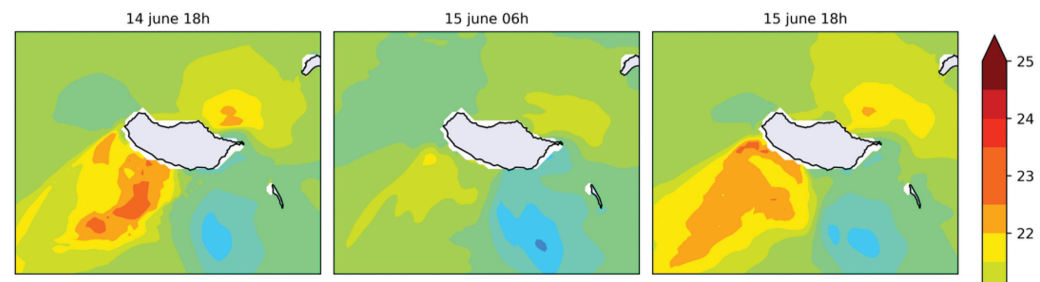

14 june $18 \mathrm{~h}$
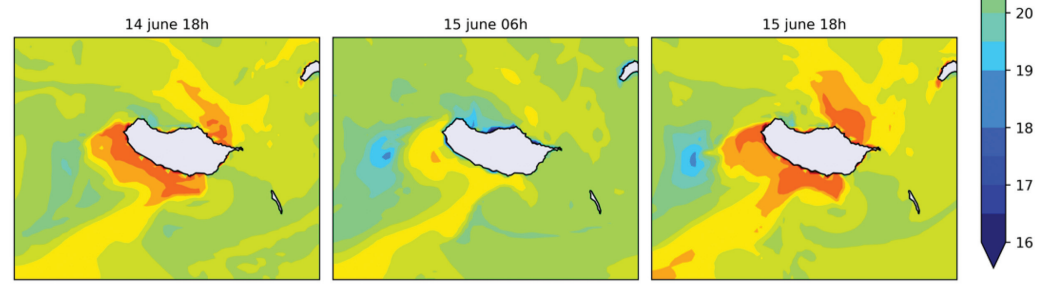

(b)
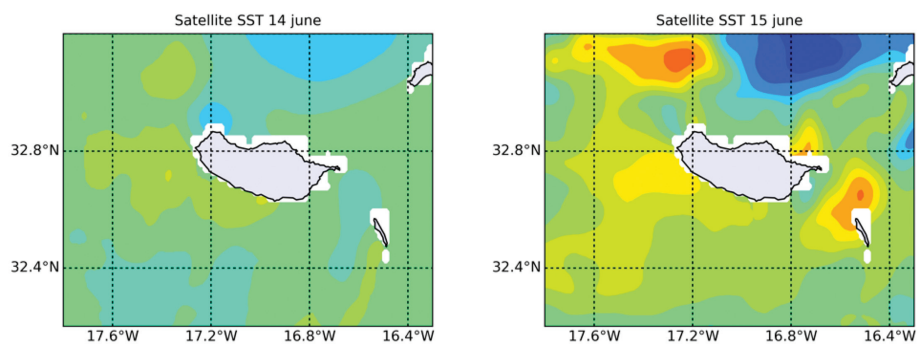

Figure 2. Sea surface temperature surrounding Madeira Island, North Atlantic for 14-15 June 2011 using two different coupled models: Coupled Ocean/Atmosphere Mesoscale Prediction System (COAMPS), and Coupled-Ocean-Atmosphere-Wave-Sediment-Transport System (COAWST) (top), as well as multisensor daily composite from Group for High Resolution Sea Surface Temperature (GHRSST) (1 km) (bottom).

coupling (Section 4a), coupling frequency and complex land-sea gradients (Section $4 b$ ), and sensitivity to grid resolution (4c). Section 5 follows with illustrations of medium-term ( $\sim$ multiple weeks) sensitivities including the impact of temporal frequency of exchange (Section 5a) and role of ocean current feedback (Section 5b). Throughout, we focus on challenging geographical settings and processes where energetic air-sea coupling is manifest. Our examples span novel hurricane genesis off Brazil and tropical weather patterns in the Maritime Continent. We employ both operationally-oriented (COAMPS) and community model (ROMS/WRF) implementations. We arrive in Section 6 at a discussion of some of the challenges facing coupled modeling systems as they strive for enhanced realism in their predictions. Section 7 contains the summary. 


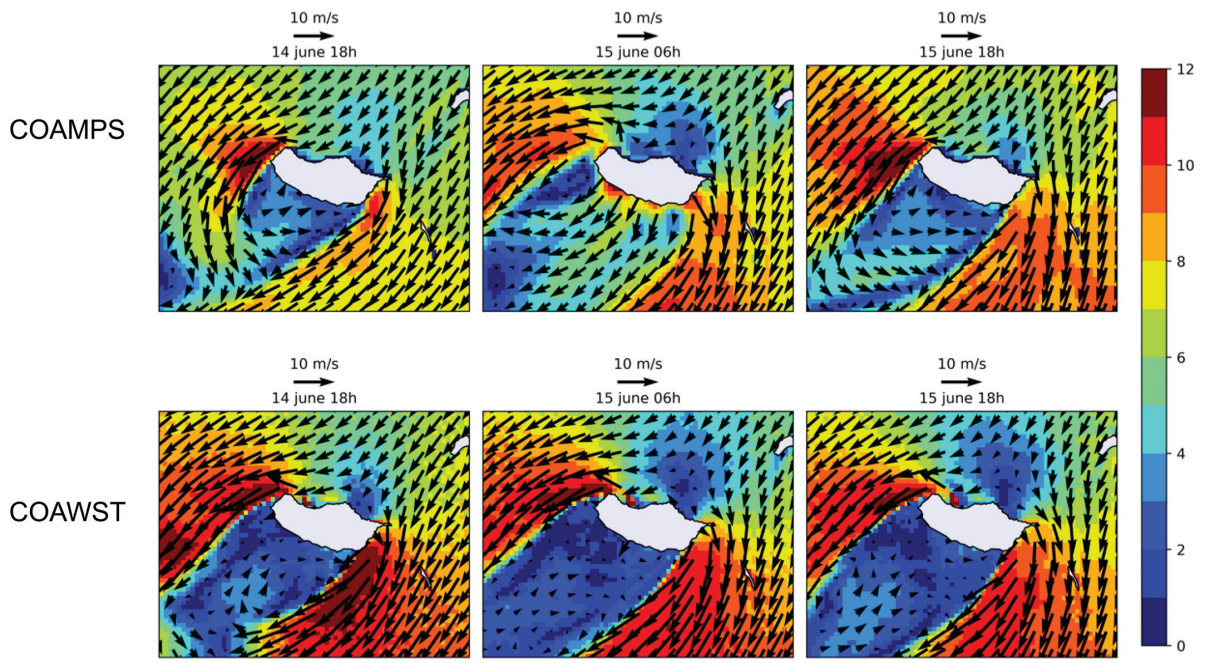

Figure 3. 10-m winds around Madeira Island, North Atlantic for 14-15 June 2011 using two different coupled models: Coupled Ocean/Atmosphere Mesoscale Prediction System (COAMPS), and Coupled-Ocean-Atmosphere-Wave-Sediment-Transport System (COAWST).

\section{Coupling framework}

\section{a. Components}

Coupled modeling systems comprise multiple components consisting of numerical atmospheric, oceanographic, and static databases and data assimilation systems for both the atmosphere and ocean (Figure 4). They can also incorporate hydrology and wave models. Typical systems have the ability to configure and cycle regional/analysis models in singlemodel (atmosphere, ocean, or wave) or coupled-model (atmosphere-ocean, ocean-wave, atmosphere-wave, and atmosphere-ocean-wave) modes.

The systems consist of databases that provide terrain, bathymetry, land-sea masks, and sources of variability such as tides, rivers, and boundary forcing and initialization fields. Data assimilation approaches for the ocean or atmosphere, or a combination, are important drivers of enhanced predictability. They are commonly utilized in operational systems but may not appear in research simulations. In the physical modules, the air and sea components exchange key parameters: 10-m surface winds, atmospheric pressure, relative humidity, atmospheric and oceanic surface temperature, precipitation, cloud fraction, surface wind stress, heat fluxes, and net shortwave and longwave fluxes. The wind and wave components share near-surface wind and Charnock parameter, and the ocean and wave components share sea surface height, surface current, Stokes drift current, wave radiation stress gradient, and bottom orbital wave current. These variables and their sources within the physical module calculations are discussed as follows and in Section 3. 


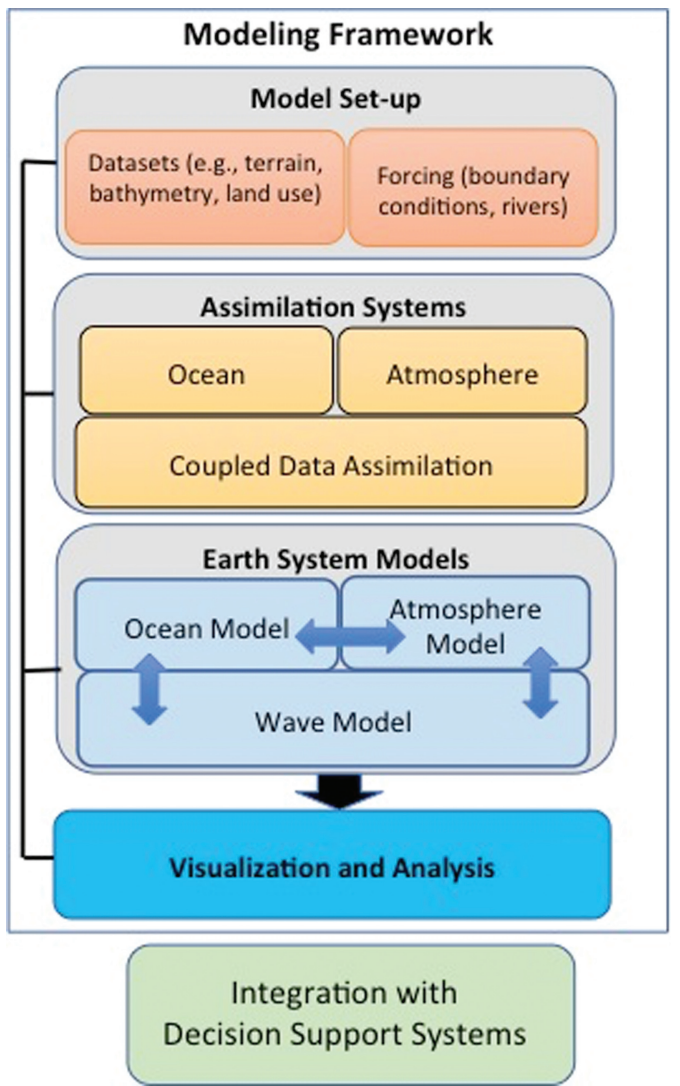

Figure 4. Schematic of a typical coupled modeling system.

In the Appendix, we use the example of two models, COAMPS and COAWST, to illustrate representative configurations of coupled systems. Details for other coupled model components may vary, but the underlying coupling strategies and functional modules remain the same. Other coupled models referenced in this paper are SCOAR (Scripps Coupled OceanAtmosphere Regional Model with ROMS/WRF) utilizing an independent flux coupler, and Unified Wave INterface Coupled Model (UWIN-CM) consisting of WRF/Hybrid Coordinate Ocean Model (HYCOM) employing ESMF. Next we examine temporal and spatial considerations in coupled modeling configurations.

\section{b. Temporal exchange}

Fluxes may be computed sequentially at a specified coupling interval or interactively at each time step. The former case requires time-averaging the fields of interest (either the surface variables or the internally computed fluxes) for consistency changes in the dynamics 
could occur if the coupling interval is larger than an hour or so. The atmosphere and ocean models alternate their time-stepping procedures in between the coupling intervals. Longterm (climate-scale) simulations often use 3-hourly, 6-hourly, or daily coupling frequency, whereas shorter than 1-hour coupling frequency is preferred for short-term simulations (e.g., for simulation of tropical cyclones and weather forecasting). In Sections 4 and 5, we study the effects of coupling frequency on short- and medium-term forecasting.

\section{c. Grid resolution}

Care must be taken at land-sea boundaries to map only quantities and fluxes influenced by underlying ocean variables rather than land variables, or excessive high fluxes can result from interpolation across the boundary.

The regridding error can be large near steep orography, complex coastlines, and small islands. To eliminate this issue, many models employ identical horizontal resolutions in the ocean and atmosphere with matching grids and land-sea mask (e.g., Seo et al. 2008b, 2014, 2016). Despite the higher computing cost of increasing the resolution of the atmosphere to match that of the ocean, one obvious benefit for the study of air-sea interaction is that the effect of air-sea coupling is maximized at the scale of the SSTs simulated by the ocean model. It also helps to lessen, somewhat, the computing burden associated with regridding, especially for large grids. In Section 4 we study coupled modeling sensitivity to resolution and land-sea gradient effects.

\section{Coupling physics}

a. Heat fluxes

Besides employing high-fidelity models of the atmosphere and ocean, the computation of the surface fluxes of heat, momentum, and freshwater is the most critical aspect of the regional and global coupling system.

The surface latent and sensible heat fluxes are very sensitive to the SST, both for its direct effect on the air-sea temperature and the saturation humidity (Cayan 1992) and for its influence on the stability of the atmospheric boundary layer (see review by Small et al. 2008) This is manifest directly in the boundary layer (BL) model of the atmosphere and in computations of latent and sensible heat fluxes computed from bulk formulae (e.g., Fairall et al. 1996). Latent heat flux, $L H=\rho_{a} L_{e} \overline{w^{\prime} q^{\prime}}$, and sensible heat flux, $S H=\rho_{a} c_{p a} \overline{w^{\prime} T^{\prime}}$, can be calculated using the standard bulk expressions $L H=\rho_{a} L_{e} C_{e}\left(q_{s}-q_{a}\right)\left|U_{a}-U_{o}\right|$ and $S H=\rho_{a} c_{p a} C_{h}\left(T_{s}-T_{a}\right)\left|U_{a}-U_{o}\right|$, respectively. The exchange coefficients, $\mathrm{C}_{\mathrm{e}}$ and $\mathrm{C}_{\mathrm{h}}$, are larger when the air overlies warm SST compared to cold SST (increasing the heat flux), although their contributions are known to be small when compared with the increased wind speeds and temperature (humidity) differences (e.g., Liu et al. 1979).

There are generally two ways to construct the flux coupler, using either bulk formula or using fluxes computed from the atmospheric model. Both methods use the atmospheric fields as the input: short- and long-wave radiation fluxes from the radiation scheme, cloud 
(a) LH WRF

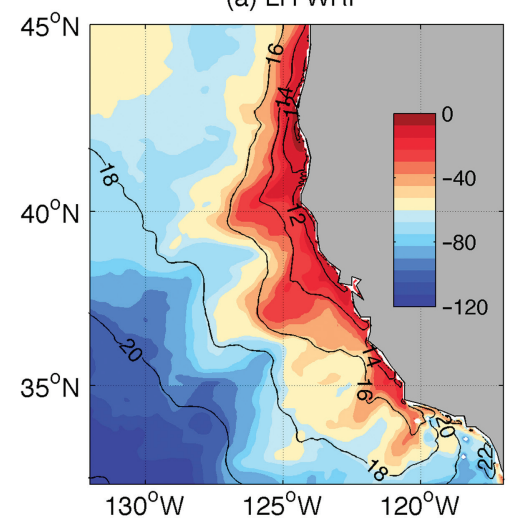

(b) LH Bulk Formula

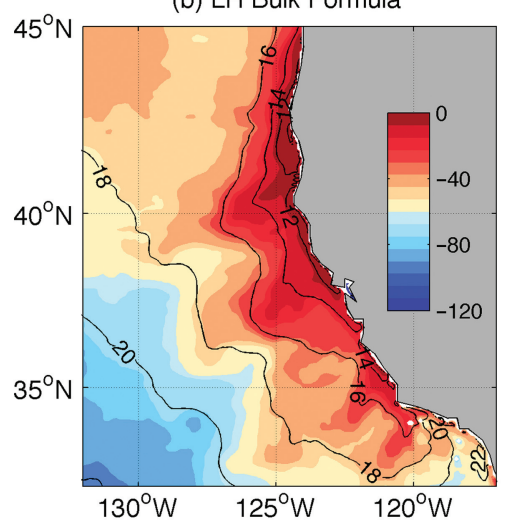

Figure 5. Two estimates of summertime (July-September) surface latent heat flux (LH) (shading,

$\left[\mathrm{Wm}^{-2}\right]$, negative cooling the ocean) averaged for 6 years (2005-2010) obtained from the 7-km Scripps Coupled Ocean-Atmosphere Regional Model (SCOAR) model (Regional Ocean Modeling System-Weather Research and Forecasting [WRF-ROMS]) simulations for the U.S. West Coast. (a) LH estimated from the WRF surface layer scheme based on MM5 Monin-Obukhov similarity in conjunction with the Yonsei University (YSU) nonlocal-K scheme (Hong, Noh, and Dudhia 2006). (b) LH estimated from the Coupled Ocean-Atmosphere Response Experiment (COARE) bulk airsea flux algorithm (Fairall, Kepert, and Holland 1994; Fairall et al. 2003). Black contours, identical in both panels, denote the climatological summertime sea surface temperature (SST) simulated by the model.

fraction from the microphysics scheme, and precipitation rate from the convection and microphysics scheme. Both methods also need the near-surface meteorological fields, such as air temperature and humidity at $2 \mathrm{~m}$ and vector and scalar wind at $10 \mathrm{~m}$, that are calculated in the planetary boundary layer (PBL) scheme, which is model dependent. These fields provided by the atmospheric model are combined with the SST predicted by the ocean model and the wave fields (if coupled to a wave model) to compute the surface latent and sensible heat, freshwater, and momentum fluxes. The two methods differ in their simplification of the surface layer process to compute the surface fluxes. The bulk formulae are based on a local closure-scheme, whereas the boundary layer model of the atmosphere invokes often complicated nonlocal PBL parameterization schemes over the land and the water (e.g., Hong and Pan 1996), which interact with the surface layer scheme to compute the surface fluxes.

The resultant surface flux estimates from the two methods can differ. Figure 5 illustrates this by comparing the estimates of the summer mean surface latent heat flux from the 7km SCOAR (WRF-ROMS) coupled model for the U.S. West Coast (Seo et al. 2016). One (left) is calculated from the WRF surface layer scheme based on the MM5 Monin-Obukhov similarity in conjunction with the Yonsei University (YSU) nonlocal-K scheme (Hong, Noh, and Dudhia 2006), and the other (right) is from the Coupled Ocean-Atmosphere Response 
Experiment (COARE) bulk air-sea flux algorithm (Fairall, Kepert, and Holland 1994; Fairall et al. 2003). The near-shore upwelling zone coincides with the region of weak latent heat flux (negative meaning cooling of the ocean), where the difference in the two latent heat flux estimates is small. However, for offshore regions with strong wind speed and warm SST, the difference can be quite large (up to $40 \mathrm{Wm}^{-2}$ ).

These differences can be due to several factors. Bulk formulae are empirical methods originally developed for the tropical oceans. The bulk formulae thus include parameterizations for marine boundary processes that are not represented in many atmospheric BL models, including sea spray effects (which influence heat fluxes), skin temperature effects, or rainfall effects on surface fluxes, modification of drag coefficients under high- and lowwind regimes, etc. (Fairall, Kepert, and Holland 1994; Fairall et al. 2003). In oceanic regions where such effects might be important to improve the accuracy of surface flux estimates, bulk formulae might be preferred. Additional advantages of using bulk formulae may derive from the flux being calculated on the ocean grid, which usually has higher horizontal resolution than the atmospheric model. The resultant surface fluxes would thus better reflect the fine-scale SST patterns, which are otherwise smoothed in the coarser atmospheric grids at which the BL models compute the surface fluxes. Bulk formulae are simple to modify in order to be used in a variety of oceanic settings, including the polar oceans (e.g., Hunk and Holland 2007). However, the surface flux directly calculated from the BL models might provide more internally consistent surface fluxes to the system (including land and ocean), thereby conserving total enthalpy and mass.

Computationally, the bulk formulaic method is more straightforward to calculate fluxes and apply to the coupler, because atmospheric variables from the BL models can be interpolated (if needed) to a common grid and a simple computation of flux components obtained with a standard scheme. The BL method instead invokes complicated nonlocal parameterizations in the atmospheric model (e.g., Hong and Pan 1996), with SST, surface velocity, and roughness (wave heights) specified as inputs at the surface from the ocean model. The resulting fluxes of momentum, sensible heat, and latent heat are then mapped to the ocean grid (if needed) as forcing terms.

Long-wave radiative fluxes are determined directly from the black body formulation for SST, whereas short-wave (incoming) radiative fluxes are computed based on the solar angle and cloudiness of the atmospheric model from the radiation and cloud microphysics schemes.

\section{b. Momentum transfer}

Turbulence is generated by the vertical shear of near-surface wind over water and land and is typically expressed as the Reynolds stress, $\tau_{x}=-\rho \overline{u^{\prime} w^{\prime}}$ and $\tau_{y}=-\rho \overline{v^{\prime} w^{\prime}}$, where prime denotes the turbulent fluctuation, overbar is the time average, and $\rho$ is the air density. A velocity scale $\mathrm{u}_{*}$, friction velocity, is derived from $u_{*}=\frac{1}{\rho} \sqrt{\tau_{x}^{2}+\tau_{y}^{2}}$. Using $\mathrm{u}_{*}$, semiempirical distribution of wind speed at height $\mathrm{z}$ within the surface layer can be determined by 
$u(z)=\frac{u_{*}}{k}\left[\ln \left(\frac{z-d}{z_{0}}\right)-\psi_{m}(z / L)\right]$, where L is the Monin-Obukhov length-scale and $\psi_{m}$ is an empirical function for stability correction. The roughness length, $\mathrm{z}_{0}$, is a parameter that accounts for the effect of the overlying wind on surface roughness due to waves (swells, gravity and capillary waves) (Bourassa, Vincent, and Wood 1999) and is often determined by the steepest waves (Taylor and Yelland 2001). This information can be obtained from a wave model (if coupled), which provides the wave-induced roughness length to the surface layer scheme to calculate the surface fluxes. If not, $\mathrm{z}_{0}$ is prescribed with a typical value of $2 \times 10^{-4} \mathrm{~m}$ over water.

Surface layer schemes, whether from the atmospheric model scheme or bulk formulae, are based on the surface layer similarity theory and solve for $\mathrm{u}_{*}$ to derive the momentum fluxes. In this formulation, local SST anomalies can modify the stability parameter, $\psi_{m}$, and thus the well-known logarithmic wind profiles within the surface layer, yielding the increased (decreased) wind speeds over warm (cold) SST (e.g., Sweet et al. 1981; Businger and Shaw 1984; Hayes, McPhaden, and Wallace 1989; Wallace, Mitchell, and Deser 1989; Chelton et al. 2004; Xie 2004).

For very fast ocean waves and transient SST conditions (e.g., mixed-layer eddies and fronts), the stability function profiles described using Monin-Obukhov similarity theory may not apply in these conditions, and the relationship between SST and surface wind may not be valid by the current empirical formulation in the surface layer schemes. Moreover, different empirical formula in BL and bulk models would also mean that there exists uncertainty in estimates of surface flux, which can impact the result of the simulation. For example, different roughness length schemes (Bao et al. 2000) produce diverse results in the intensity of Hurricane Opal (1995).

The momentum fluxes (stresses) at the air-sea interface have traditionally been computed from wind speed, while assuming the ocean is at rest (Bye 1986). However, modern computations of the stress can easily accommodate a changing ocean surface current and waves in both the BL formulation and the bulk formulaic calculation of fluxes. In the former case, coupling of the atmospheric model to a wave model can provide the important surface roughness length scale associated with the wave heights. Surface currents, by creating a relative motion between air and sea surface, modify the total wind stress, $\tau=\rho C_{d}\left|U_{a}-U_{o}\right|\left(U_{a}-U_{o}\right)$, where $U_{a}\left(U_{o}\right)$ refers to the wind (surface current) speed. Although in most cases surface current speed is an order of magnitude smaller than the wind speed, the influence of the ocean currents associated with the mesoscale eddies and swift surface currents on the wind stress have been noted in multiple studies (e.g., Kelly et al. 2001; Cornillon and Park 2001; Park, Cornillon, and Codiga 2006). Several of these studies showed that an inclusion of surface currents in the wind stress formulation leads to a decrease in the mean and eddy kinetic energy (Pacanowski 1987) due in part to increased surface drag (Seo et al. 2007; Small et al. 2009; Eden and Dietze 2009; Seo et al. 2015; Seo 2017). Furthermore, Ekman pumping is sensitive to surface vorticity and its gradient (Stern 1965; Gaube et al. 2015), implying some significant impact on the energetics of the eddy and circulation (Seo et al. 2015) and biological productivity (Chelton et al. 2011; Gaube et al. 2014). 


\section{c. Freshwater fluxes}

The net freshwater fluxes are the sum of evaporation $(\mathrm{E})$, precipitation $(\mathrm{P})$, and runoff (R) and are important for the atmospheric moisture budget and convective response. The freshwater fluxes also affect the upper ocean salinity and density, which then affect deep ocean convection and subduction. Evaporative fluxes to the atmosphere are directly derived from the latent heat computation in the BL model or the bulk formulaic parameterization. Precipitation is obtained from the atmospheric model based on the cumulus scheme for convective rain and the microphysics scheme for nonconvective rain.

In addition, the river discharge is often an important driver of the regional ocean circulation and the hydrological cycle such as in the Bay of Bengal in the Indian Ocean (Seo et al. 2009) and the western equatorial Atlantic near the Amazon River mouth (Hu et al. 2004). River runoff has been relatively crudely treated in regional ocean and coupled models, often prescribed as the local source of freshwater (such as precipitation as in Howden and Murtugudde 2001), as the virtual runoff (e.g., Yu and McCreary 2004), or by prescribed salinity stratification (e.g., Vialard and Delecluse 1998). However, the runoff information can now be provided from the land model of the atmospheric component at a specified location of the domain. This approach ensures the conservation of the freshwater content in the system. Although the freshwater fluxes affect density, the mass changes in precipitation and evaporation in the oceanic part of regional coupled models are very small and therefore often ignored in the model formulation.

A new development in coupled models is to introduce a short-term hydrology forecast capability into the coupled modeling suite. For example, hydrological (WRF-Hydro) and enhanced soil treatment (NASA Land Information System [LIS]) are being added to COAMPS to improve prediction of coupled air-sea-hydrological processes. The benefit of representing inland precipitation via an atmospheric model accrues to the hydrology model, which then affords the forecasting of river discharge at the coast. Significant buoyancy effects from river discharge are apparent in many coastal regions, and prediction of the local impacts of flood hazards and storm surge are a growing and significant application area of coupled models (Saleh et al. 2016).

\section{Short-term forecasting (days to week) applications}

\section{a. Effects of wave coupling}

In short-term forecasting applications, a key consideration is whether wave coupling can increase the fidelity of the simulation. In Section $3 \mathrm{~b}$, the role of momentum transfer in the physics of coupling was emphasized. Here we examine U.S. east coast circulation during a hurricane and evaluate the impact of waves in the context of air-sea coupled modeling. Such an evaluation approach can also be employed to assess the value of coupling other components of the earth system (e.g., hydrology, ice) for the particular region of interest.

Feedback from the ocean model component to the wave model component typically includes the input of ocean model surface currents and water levels to the wave component. 
Water levels can modify the water depth used within the wave model physics calculations, though this effect is only significant if the water depth is sufficiently shallow that the waves feel the ocean bottom. Surface currents input into the wave model alter the effective wind speed (i.e., the wind speed relative to a frame of reference moving with the currents) and the horizontal shear of the currents produce changes in the length, height, and direction of the waves.

Wave model feedback to the ocean model component typically includes the Stokes' drift current (SDC), the wave radiation stress gradients, and the characteristic velocity and frequency of the wave orbital motion near the ocean bottom. Wave motion near the bottom is used to enhance bottom drag in shallow water, and the radiation stress gradients that arise from breaking waves and their associated momentum in shallow water are applied to the ocean model as a surface stress. The SDC is the average current velocity when following a specific fluid parcel as it travels with the fluid flow. For instance, a particle floating at the free surface of water waves, experiences a net Stokes drift current in the direction of wave propagation. The SDC is calculated within the wave model component and passed to the ocean model component to advect the ocean model fields. The SDC may also be used in the enhancement of vertical mixing within the ocean model by Langmuir turbulence (Kantha and Clayson 2004). Additional shear production terms are added to the turbulent kinetic energy equations within the ocean model component to enhance vertical mixing. The following are some examples of air-ocean-wave model interactions that improve model skill.

For the northeastern Gulf of Mexico outer continental shelf and slope domain considered here, observational wave and ocean data sets included an array of 14 Acoustic Doppler Current Profilers (ADCP) deployed by the Naval Research Laboratory (Teague et al. 2007) in May 2004 (Figure 6). These data were utilized by Smith et al. (2013) in relation to atmosphere-ocean-wave coupling in Hurricane Ivan using COAMPS-TC without a wave component.

The COAMPS-TC forecast track and intensity exhibited low errors allowing for a reasonable comparison of the ocean and wave fields. Table 1 depicts summary statistics for six shallow-water ADCPs (M1-M6) shown in Figure 6 averaged over a 72-hr forecast period initiated on 14 September 2014, 1200 UTC. The complex correlation coefficient (see Smith et al. 2013) accounts for both the current speed and direction. The table shows highly correlated speeds and directions for all 6 ADCP's with the mean directional error lowest for the 3 northern-most locations with ranges of approximately 10 to $15^{\circ}$ for M4-M6 to the south. The modeled speeds agree well with observations. Figure 7 shows a comparison of Navy Coastal Ocean Model (NCOM) current magnitude versus observation at the location of ADCP M1. The NCOM results were adjusted temporally by six hours to more closely match the timing of the hurricane track. The COAMPS-TC simulation was approximately six hours too fast.

In order to address these discrepancies, a fully coupled atmosphere-ocean-wave hindcast study was performed for a 2-month period beginning 1 September 2013. Figure 8 (top) depicts the modeling domain with the location of two NDBC (National Data Buoy Center) 


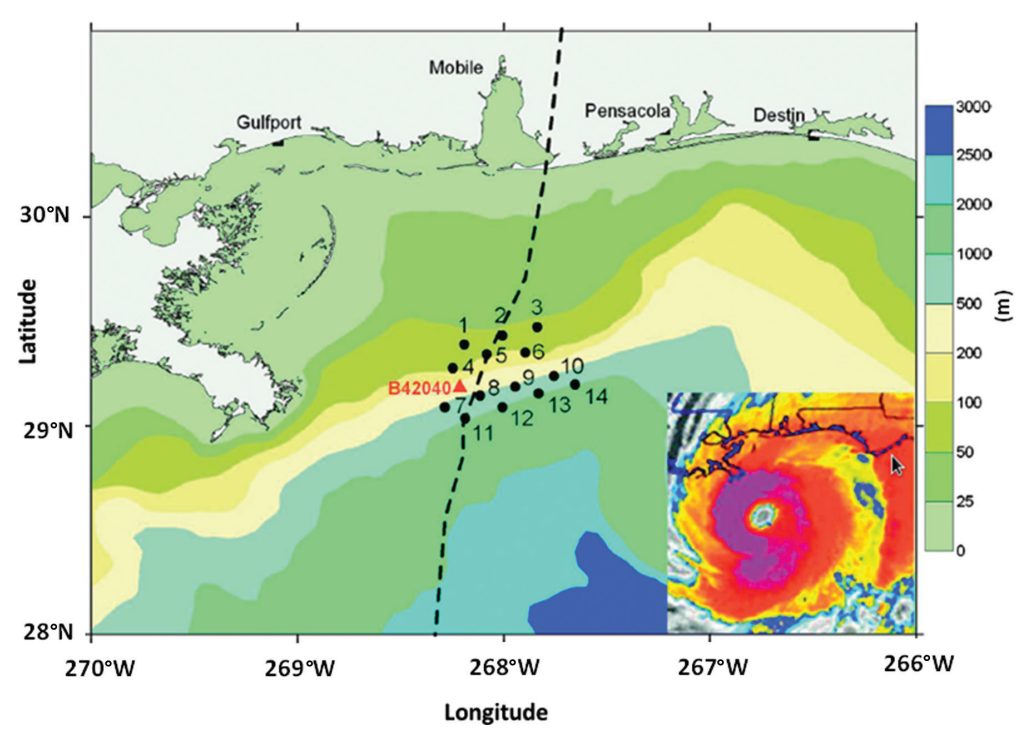

Figure 6. Acoustic Doppler Current Profilers (ADCP) array in the northern Gulf of Mexico (adapted from Teague et al. 2007). Bathymetry contours (m) and the best track of Hurricane Ivan is shown. Inset: Infrared satellite image of Hurricane Ivan as it approaches the northern Gulf of Mexico coast on 15 September 2004.

Table 1. Acoustic doppler current profiles (ADCP) versus NCOM current statistical comparisons for ADCPs M1-M6 averaged over the 72-hr 1200 UTC 14 September 2004 forecast (Smith et al. 2013). CCC, complex correlation coefficient (takes in account speed and direction [see Kuzmic et al. 2006]); MDE, mean directional error; MB, mean bias; OBS, observed current speed.

\begin{tabular}{lcccccc}
\hline ADCP & $\begin{array}{c}\text { Top bin } \\
\text { depth }(\mathrm{m})\end{array}$ & CCC & $\begin{array}{c}\text { MDE } \\
(\text { degrees })\end{array}$ & $\begin{array}{c}\text { Speed } \\
\text { MB }\left(\mathrm{ms}^{-1}\right)\end{array}$ & $\begin{array}{c}\text { Max Speed } \\
\text { NCOM }\left(\mathrm{ms}^{-1}\right)\end{array}$ & $\begin{array}{c}\text { Max Speed } \\
\text { OBS }\left(\mathrm{ms}^{-1}\right)\end{array}$ \\
\hline M1 & 6 & 0.83 & 1.57 & 0.046 & 2.04 & 2.14 \\
M2 & 4 & 0.77 & 8.45 & 0.082 & 1.75 & 1.87 \\
M3 & 6 & 0.79 & 12.29 & 0.110 & 1.61 & 1.73 \\
M4 & 10 & 0.78 & 10.44 & 0.121 & 1.77 & 1.96 \\
M5 & 11 & 0.82 & 10.37 & 0.071 & 1.82 & 1.91 \\
M6 & 9 & 0.83 & 15.54 & 0.145 & 1.69 & 1.82 \\
\hline
\end{tabular}

buoys used to evaluate the system in coastal waters. The ocean and wave models had a grid resolution of $2 \mathrm{~km}$ and were fully coupled to a 6-km atmospheric model. Initial and boundary conditions were provided by the models described in the Appendix. The model was spun-up for a 14-day period and run from 1 September to 27 October 2013. The wave model had a directional resolution of $7.5^{\circ}$ (48 bins) and 25 frequencies. NCOM simulations included tides and data assimilation. In this study, we will only present results for the Cape Henry, VA buoy location. Model results for Duck, NC (not shown) were very similar. 
(a)

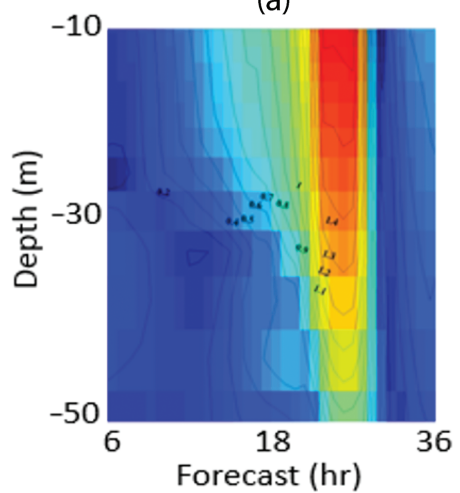

(b)

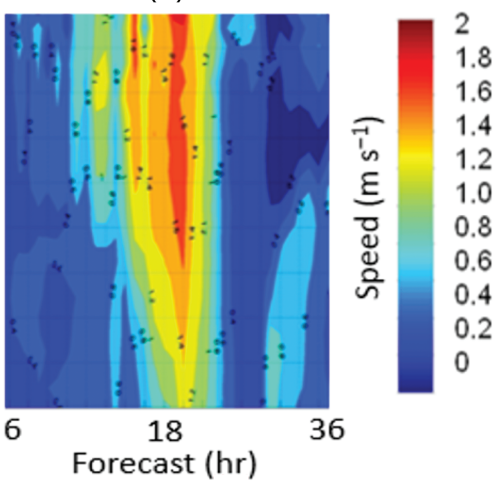

Figure 7. Current velocity vertical profile for Navy Coastal Ocean Model (NCOM) (left) and observations (right) at the location of acoustic Doppler current profiler (ADCP) M1, modified from Smith et al. 2013.

Figure 8 (bottom) depicts a model-data comparison of significant wave heights at Cape Henry, VA (44099) during September/October 2013. The red line shows the significant wave heights with the fully coupled model, the blue line represents the wave-only results, and the black dashed line depicts the observed wave height. Several events are identified where the observed wave height exceeds 2 meters. The highest wave height was recorded on October 11 when it surpassed $4 \mathrm{~m}$. This is associated with a low-pressure system located just off the North Carolina coast with a minimum central pressure of 1,007 hPa. This feature was resolved well by the COAMPS atmospheric model (not shown). Table 2 presents summary statistics for the comparisons between the uncoupled and coupled wave model at this location.

Overall, the wave results from the coupled model simulations perform better than the wave-only runs. This is evident during periods of peak wave events (e.g., 11 October storm) where the uncoupled model wave heights gradually decrease, whereas the coupled model portrays a more rapid decline in wave heights and strong agreement with observations. The wave period biases were lower in October and within the range of instrument accuracy for both sets of hindcasts. The wave directions for the coupled run show improved skill with a bias of $-6.3^{\circ}$ versus $-11.5^{\circ}$ and within the directional resolution of $7.5^{\circ}$ for Simulating WAves Nearshore (SWAN) (and instrument accuracy of $10^{\circ}$ ). In summary, the inclusion of currents (wave-current interaction) provides a more accurate wave-height field and is well justified in significant sea states.

\section{b. Coupling frequency and complex land-sea gradients}

Of key importance for coupled model configurations is the choice of frequency of model coupling (in time), as mentioned in Sections 1a and 2b. Furthermore, in complex coastal 


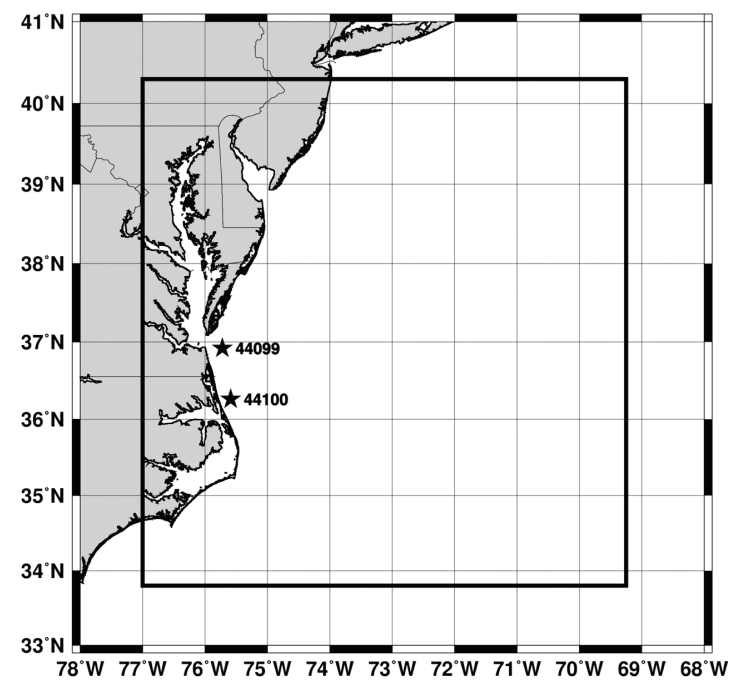

(a)

Cape Henry (44099)

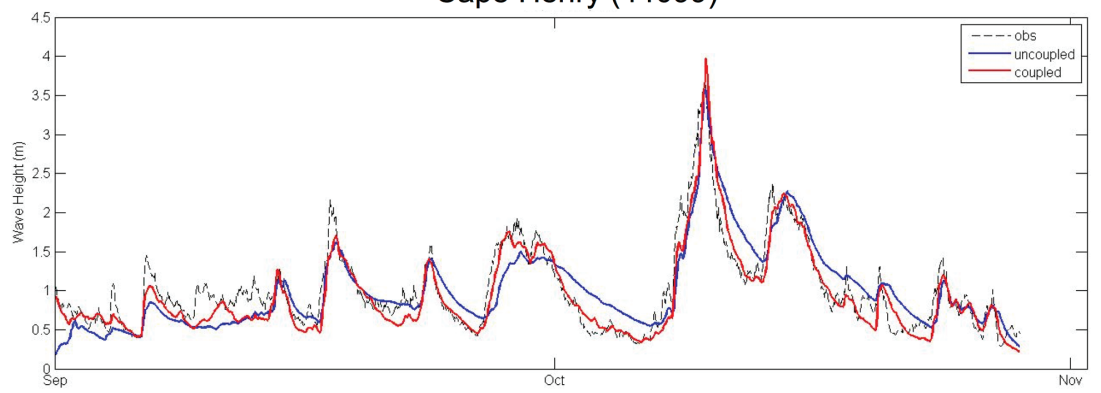

Figure 8. (top) Bounding box indicates the east coast U. S. domain of the fully coupled atmosphereocean-wave COAMPS (Coupled Ocean/Atmosphere Mesoscale Prediction System). The atmospheric model was run at 6-km resolution and the ocean and wave models were run at 2-km resolution. National Data Buoy Center (NDBC) buoy 44099 (Cape Henry) is shown near the mouth of Chesapeake Bay. (bottom) Comparison of significant wave height between coupled (red), uncoupled (blue), and observed (dashed black) wave height at Cape Henry, VA for the period of 1 September to 27 October 2013.

regions with steep terrain, the diurnal cycle depends on land-sea gradients and can be a challenge to simulate accurately. One example of such a setting is the Maritime Continent (MC) that bridges the Indian and Pacific Oceans.

The MC is characterized by strong multiscale (diurnal to intraseasonal) and multicomponent (land, atmosphere, and ocean) interactions that are not properly represented in many global prediction models. The subdaily variability in rainfall, and wind initiated over steep orography interacts with the adjoining coastal oceans to influence a region's intraseasonal rainfall variability, and the Madden-Julian Oscillation (MJO). Prediction models show that 
Table 2. Statistics showing comparison of wave height, period, and direction for September and October 2013 between NDBC (National Data Buoy Center) Buoy 44099 versus SWAN for uncoupled (left) and coupled air-ocean-wave (right) hindcasts. Shaded boxes indicate improved results over the coupled or uncoupled runs. RMSE, root-mean squared error; $\mathrm{MB}$, mean bias; CC, correlation coefficient.

\begin{tabular}{lccccccc}
\hline & \multicolumn{3}{c}{ Uncoupled } & & \multicolumn{3}{c}{ Coupled } \\
\cline { 2 - 3 } & MB & RMSE & CC & MB & RMSE & CC \\
\hline September 2013 & & & & & & \\
Wave height (m) & -0.15 & 0.28 & 0.75 & -0.12 & 0.20 & 0.90 \\
Wave period (s) & -1.64 & 3.5 & 0.47 & -1.46 & 2.80 & 0.55 \\
Wave direction ( $\left.{ }^{\circ}\right)$ & -7.51 & & 0.45 & -9.08 & & 0.49 \\
October 2013 & & & & & & & \\
Wave height (m) & 0.11 & 0.33 & 0.90 & -0.04 & 0.22 & 0.95 \\
Wave period (s) & -0.35 & 0.60 & 0.80 & -0.71 & 0.97 & 0.81 \\
Wave direction $\left(^{\circ}\right)$ & -11.54 & & 0.59 & -6.28 & & 0.68 \\
\hline
\end{tabular}

extended-range MJO prediction skill drops sharply as the MC is approached, suggesting that some aspects of the deep convection over the MC are underrepresented. On the MC, thermally forced diurnal convection over the land is a primary factor that entails the deep convection over the ocean, often leading to a formation of mesoscale convective systems. The propagation and intensification of diurnal rainfall across the land-sea boundaries is a salient feature of the rainfall variability in the MC, which is known to explain the majority of the intraseasonal rainfall variability (Peatman, Matthews, and Stevens 2014).

The primary driver of diurnal rainfall is the diurnal land heating (e.g., Mapes, Warner, and $\mathrm{Xu}$ 2003). When averaged over the MC regions, typical amplitudes of diurnal land surface temperature (LST) and 2-m air temperatures (T2) are about $10^{\circ} \mathrm{C}$ and $4^{\circ} \mathrm{C}$, respectively, whereas diurnal SST amplitude is much less, about $0.5^{\circ} \mathrm{C}$ (Figure 9). Even this relatively weak diurnal SST, however, should modulate the land-sea thermal contrast and the landsea breezes, to which the propagation and intensification process of the rainfall is sensitive (Houze et al. 1981). Diurnal SST is not well-represented in many prediction models, and the extent to which the diurnal SST influences the diurnal amplitude and phase of the rainfall is an active area of research.

To quantify this, a comparison is made from two regional model simulations targeting the one-month (14 November-13 December 2011) period of the Dynamics of the MJO/Cooperative Indian Ocean Experiment on Intraseasonal Variability in Year 2011 (DYNAMO/CINDY) experiment. One run is based on the multiensemble SCOAR (WRFROMS) fully coupled model simulations with 1-hourly coupling frequency (hereafter CF1) to represent the realistic diurnal SST and the air-sea interaction. The other run is based on a WRF-only simulation that is identical to WRF of CF1 but forced with the daily mean SST from CF1 (CF1DM). Having the identical daily mean SST, the difference in atmospheric processes between CF1 and CF1DM indicates the effect of SST fluctuations on a diurnal 
(a) Rain Land

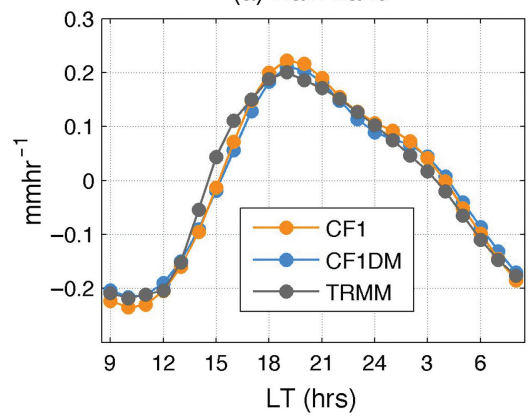

(c) LST and T2

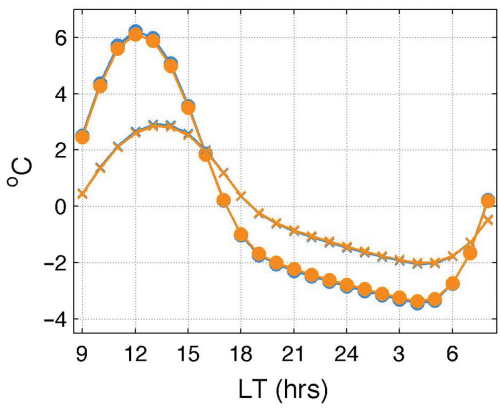

(b) Rain Ocean

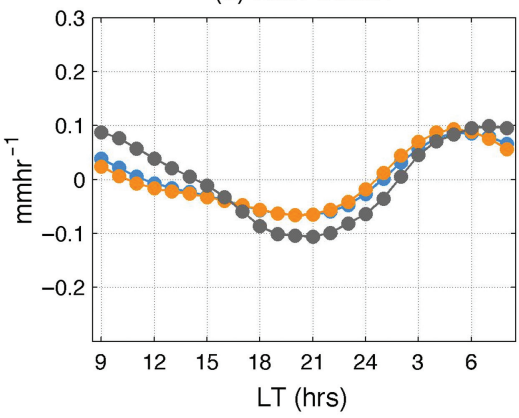

(d) SST

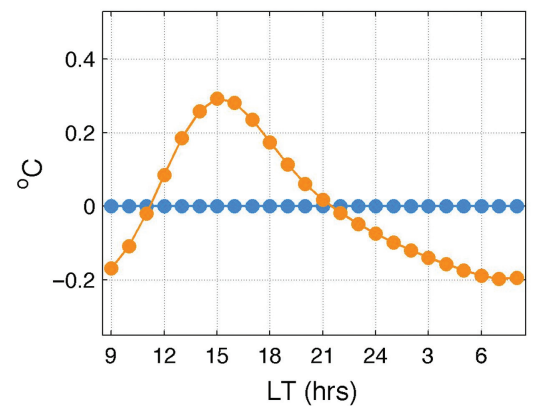

Figure 9. Hourly composites of (a) rainfall $\left(\mathrm{mmhr}^{-1}\right)$ averaged over the land only and (b) rainfall averaged over the ocean only for the area of $95^{\circ} \mathrm{E}-150^{\circ} \mathrm{E} 10^{\circ} \mathrm{S}-7^{\circ} \mathrm{N}$ for 14 November to 13 December 2011. (c) as in (a) but showing the land surface temperature (LST) $\left({ }^{\circ} \mathrm{C}\right.$, line with filled circles) and 2-m air temperature (T2; ${ }^{\circ} \mathrm{C}$, line with crosses), and (d) Sea surface temperature (SST). Rainfall is from the Tropical Rainfall Measuring Mission (TRMM)-3B42 satellite analysis product. CF1 is the fully coupled Weather Research and Forecasting-Regional Ocean Modeling System (WRF-ROMS) model simulation with diurnal sea surface temperature (SST). CF1DM is the uncoupled WRF simulation forced with the daily mean SST.

basis. Diurnal SST also raises the daily mean SST (Shinoda 2005), affecting initiation and intensity of the MJO convection (Seo et al. 2014), but this process is not considered. Because the ocean-atmosphere resolution of the model is $40 \mathrm{~km}$, it relies on parameterized convection; that is, it does not capture the gravity wave dynamics that partially explain the offshore migration of diurnal rainfall (Love, Matthews, and Lister 2011). Diurnal SST in the present model affects diurnal rainfall by altering the land-sea thermal contrasts.

Figure 9(a) and (b) compares the hourly composite anomalies of rainfall averaged separately over the land grid points and the ocean grids within the $\mathrm{MC}$ domain $\left(95-150^{\circ} \mathrm{E}\right.$, $10^{\circ} \mathrm{S}-7^{\circ} \mathrm{N}$ ). The TRMM-3B42 precipitation estimate (gray) shows that the land rainfall is initiated in the afternoon and peaks in the evening hours (19 Local Time [LT]), and the daily minimum rainfall is during the morning hours at 10LT (Mori et al. 2004). The diurnal rainfall over the ocean has weaker diurnal amplitude and roughly the opposite phase, with 
the maximum in early morning hours (9LT) and the minimum in late night (21LT). The two simulations capture reasonably well the observed amplitude and phase of the over-land and over-water diurnal rainfall variability. A close examination indicates that $\mathrm{CF} 1$ produces lower rainfall in the early morning hours (e.g., 6-10LT) compared to CF1DM both over the land and ocean, while the difference in daily maximum (minimum) rainfall over land (ocean) at 19LT is negligible. The effect of diurnal SST is most clearly seen in the early morning hours, which is when the over-water diurnal rainfall is at a maximum and over-land rainfall is at a minimum.

The difference in diurnal rainfall is attributed to the altered diurnal land-sea thermal contrast. Figure 9(c) shows the diurnal composite of LST/T2, which are nearly identical between CF1 and CF1DM. The inclusion of diurnal SST in CF1 (Figure 9[d]) produces the lower SST by $0.2^{\circ} \mathrm{C}$ in the morning/night hours but the higher SST by $0.3^{\circ} \mathrm{C}$ during the daytime. Because the early morning hours correspond to the land breeze condition, the cooler SST in CF1 implies reduced land-sea thermal contrast and the weakened land breeze. This would lead to weaker propagation of the land rainfall and the weaker rainfall over the ocean in CF1. Likewise, under the sea breeze condition during the daytime, CF1 SST is higher, weakening the sea breeze and the intrusion of relatively cool marine airmass inland. Though this coincides with the slightly stronger over-land rainfall maximum (Figure $9[a]$ ), this effect appears to be small, as the diurnal rainfall at this time is dominated by the vigorous thermal convection.

The top panel of Figure 10 shows the spatial patterns of the diurnal amplitude of rainfall $(\Delta \mathrm{r})$, calculated following Love, Matthews, and Lister (2011), by fitting the hourly composite rainfall to a curve of the form, $r=\bar{r}+\Delta r \cos 2 \pi\left(t-t_{\max }\right) / 24$. Here, $\mathrm{r}$ is the total rainfall, $\bar{r}$ the daily mean rainfall, $\mathrm{t}$ the time (in hours), $\Delta \mathrm{r}$ the amplitude of the diurnal harmonic, and $t_{\max }$ the time of maximum rainfall. The same method is applied to T2. In the observations and CF1 (Figure 10[a] and [b]), $\Delta \mathrm{r}$ is strong, reaching $1 \mathrm{mmhr}^{-1}$ over land with high elevation, whereas, in the open ocean, $\Delta \mathrm{r}$ is small and the diurnal cycle is not well-defined. $\Delta \mathrm{r}$ remains relatively strong in the coastal regions that are affected by the migration of diurnal rainfall. The percent change in $\Delta \mathrm{r}$ due to diurnal SST (Figure 10[c]) suggests that $\Delta \mathrm{r}$ increases moderately by $5 \%$ to $10 \%$ in Borneo and northern Sumatra, but it strongly decreases by more than $10 \%$ to $20 \%$ in southern Sumatra, Java, northern Borneo, and New Guinea. The change of $\Delta \mathrm{r}$ is accompanied by noticeable shifts in $\mathrm{t}_{\max }$. Figure 10(d) and (e) shows that TRMM and CF1 have the $t_{\max }$ at night and morning hours over land and at early morning over the coastal seas. The shift in $t_{\max }$ between CF1 and CF1DM (Figure 10[f]) can be up to \pm 3 hours. Diurnal SST clearly modulates the amplitude and timing of the diurnal rainfall over land.

The areas of reduced $\Delta \mathrm{r}$ coincide well with the regions of reduced diurnal temperature range. Figure $10(\mathrm{~g})$ and $(\mathrm{h})$ show that $\Delta \mathrm{T} 2(\Delta \mathrm{LST}$ as well, not shown) is large in the highlands reaching $2.5^{\circ} \mathrm{C}$ in the ERA-Interim and exceeding $3^{\circ} \mathrm{C}$ in $\mathrm{CF} 1$. In contrast, the low-lying coastal regions facing the ocean exhibit considerably weaker $\Delta \mathrm{T} 2 . \Delta \mathrm{T} 2$ increases weakly $(\sim 5 \%)$ in regions of the moderate increase in $\Delta \mathrm{r}$, whereas it strongly decreases in 
(a) TRMM $\Delta r$

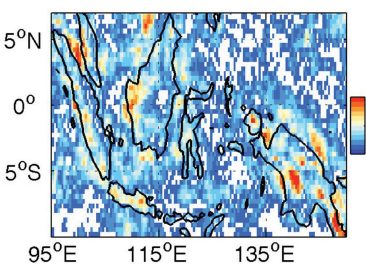

(d) TRMM Rain Tmax

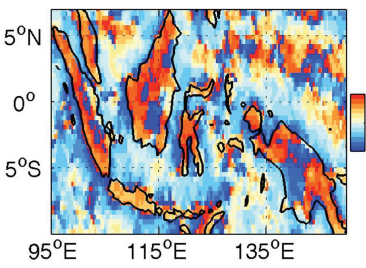

(g) ERA-I $\triangle T 2$

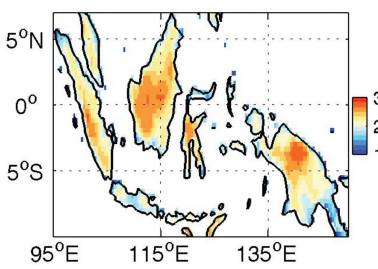

(b) CF1 $\Delta r$

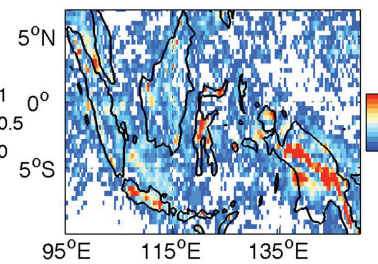

(e) CF1 Rain Tmax

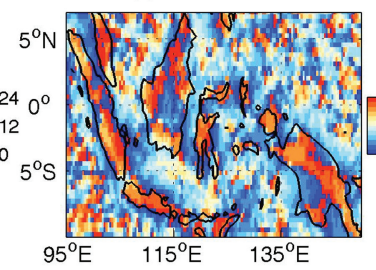

(h) $\mathrm{CF} 1 \Delta \mathrm{T} 2$

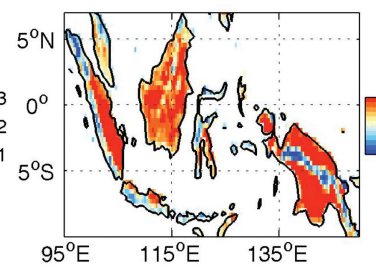

(c) CF1 and CF1DM $\Delta r \%$

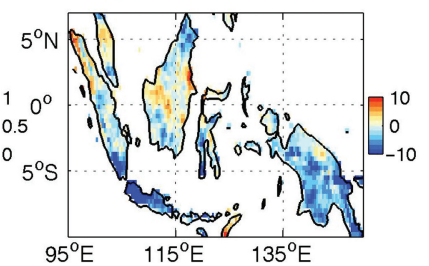

(f) CF1 and CF1DM Rain Tmax difference

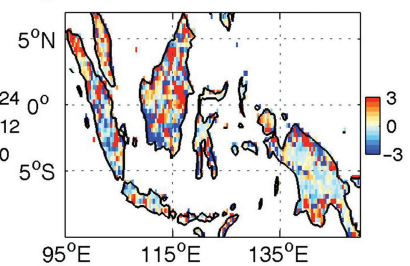

(i) CF1 and CF1DM $\Delta \mathrm{T} 2 \%$

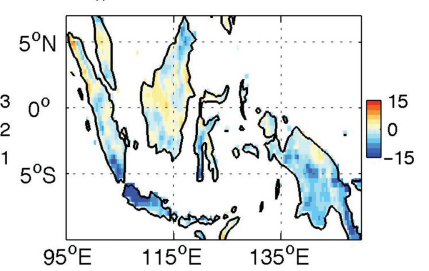

Figure 10. (top): Diurnal amplitude of rainfall $\left(\Delta \mathrm{r}, \mathrm{mmhr}^{-1}\right)$ from (a) Tropical Rainfall Measuring Mission (TRMM) and (b) CF1. (c) Percent difference over land between CF1 and CF1DM, i.e., (CF1-CF1DM)/CF1 * 100. (middle) Peak diurnal rainfall hours ( $\mathrm{T}_{\max }$ ) from (d) TRMM, (e) CF1, and (f) the difference between CF1 and CF1DM. (bottom): Diurnal amplitude of 2-m air temperature $\mathrm{T} 2\left(\triangle \mathrm{T} 2,{ }^{\circ} \mathrm{C}\right)$ from $(\mathrm{g})$ ERA-Interim, (h) CR1, and (i) percent difference between $\mathrm{CF} 1$ and CF1DM.

places where $\Delta \mathrm{r}$ is also decreased. The reduction in $\Delta \mathrm{r}$ and $\Delta \mathrm{T} 2$ are most pronounced in Java and southern New Guinea, implying that the diurnal rainfall and the diurnal temperature ranges in these regions are subject to a strong marine influence.

In summary, the analysis demonstrates some sensitivity of the amplitude and phase of the diurnal rainfall over the MC islands to diurnal SST. Incorrect phasing and amplitude of the MC diurnal rainfall simulated in prediction models can be thus partially attributable to lack, or underrepresentation, of diurnal SST. However, the result comes with an important caveat in that the model does not resolve the essential gravity wave response to convective and stratiform heating (Houze 1997). A more rigorous analysis is needed in a numerical model that explicitly resolves the diabatic heating and the gravity wave response. Nevertheless, it is evident that properly resolving with sufficient temporal resolution the land and ocean diurnal processes in the vicinity of land-sea gradients is a prime consideration in the MC. Diurnal air-sea coupling would be important for skillful simulation of SST and rainfall in 
other oceans surrounded by landmasses such as the Bay of Bengal. The strong salinitydriven stratification is known to amplify the diurnal SST (Mujumdar et al. 2011), which in turn affects the air-sea flux and diurnal rainfall variability (Weller et al. 2016; Kilpatrick, Xie, and Nasuno 2017).

\section{c. Sensitivity to grid resolution}

The role of grid resolution (Section 2c) is illustrated with an example of a novel phenomena of an intense extra-tropical cyclone that evolved into a hurricane that impacted Brazil. The choice of resolution impacts the quality of the fluxes (Section 3a) and propagates through the numerics of the simulation to manifest in the simulated hurricane track.

Brazil had its first documented experience with a hurricane during March 2004 when an extratropical storm transitioned into a hurricane (reaching category 1), named Hurricane Catarina. The diagnosis and understanding of the life cycle of this phenomenon was quite complex as an extratropical cold core low pressure system located southeast of Brazil shifted offshore over the open sea (Mctaggart-Cowan et al. 2006). It developed, gained strength, and transitioned into a tropical cyclone due to various physical factors. Beyond the dynamics and thermodynamics of the atmosphere, one of the suggested causes was the displacement of Catarina over the relatively warmer waters of the Southwest Atlantic that were present along the hurricane's trajectory (Davis and Bosart 2004; Mctaggart-Cowan et al. 2006; Vianna et al. 2010).

The oceanic surface feedback was crucial in determining the life cycle of this system. Hurricane Catarina interacted with four warm core rings, according to the oceanic observational study by Vianna et al. (2010). From that analysis it is possible to deduce that the destabilization of the marine atmospheric boundary layer, which promoted low vertical wind shear in the lower troposphere, may have played a key role in the intensification of this system, because the temperatures were higher $\left(24^{\circ} \mathrm{C}\right)$ in the warm rings than the surface air temperature $\left(14^{\circ} \mathrm{C}\right)$. This is an ideal opportunity for the transfer of heat from the sea to the atmosphere, as shown in Pezzi et al. $(2005,2009)$ in the Brazil-Malvinas Confluence (BMC) region. This mechanism is also reported in several studies of nearby ocean regions with strong thermal gradients in oceanic fronts (Acevedo et al. 2010; Camargo et al. 2013).

In order to gain understanding of the physics of Hurricane Catarina's transition from an extratropical to tropical cyclone, a total of four numerical simulations were performed. They were divided into a) atmospheric simulations with WRF-only where prescribed SST is used and b) coupled (COA), which used fully two-way coupled WRF and ROMS. Furthermore, these simulations were divided according to their horizontal resolution (20 and $6 \mathrm{~km})$. All simulations started on 18 September 2004 at 00 GMT and ran for 10 days without being reinitialized and without any flux correction.

Both WRF and COA simulate well the beginning of the phenomenon, in both 6- and 20$\mathrm{km}$ resolutions (Figure 11). For the high-resolution experiments, Catarina's lowest sea level pressure reached 998 (WRF) and $1001 \mathrm{hPa}$ (COA). For the very-high-resolution simulations, 
(a) PNM (hPa) MIN=998.395 06Z23MAR2004

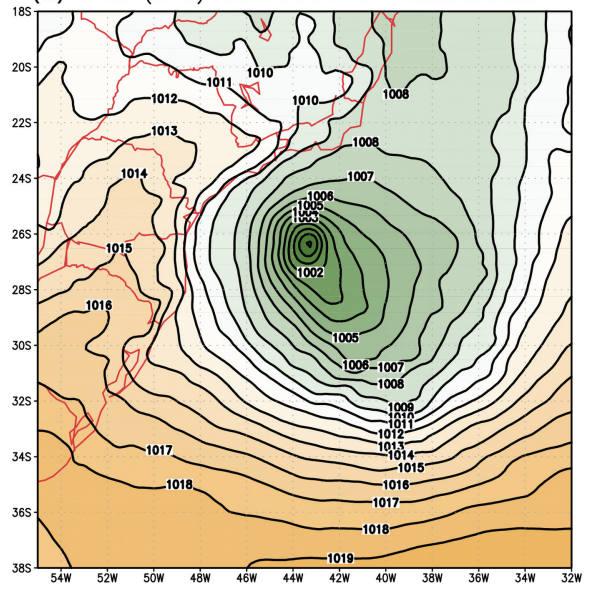

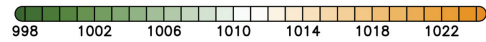

(c) PNM (hPa) MIN=1000.74 06Z23MAR2004

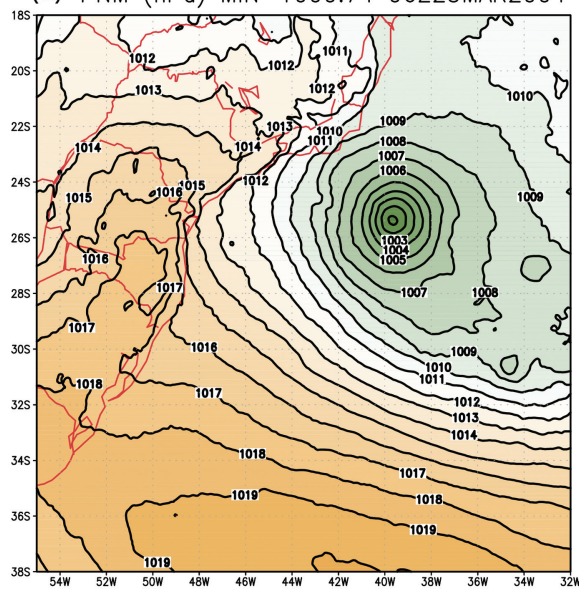

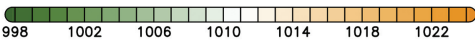

(b) PNM (hPa) MIN=1001.68 06Z23MAR2004

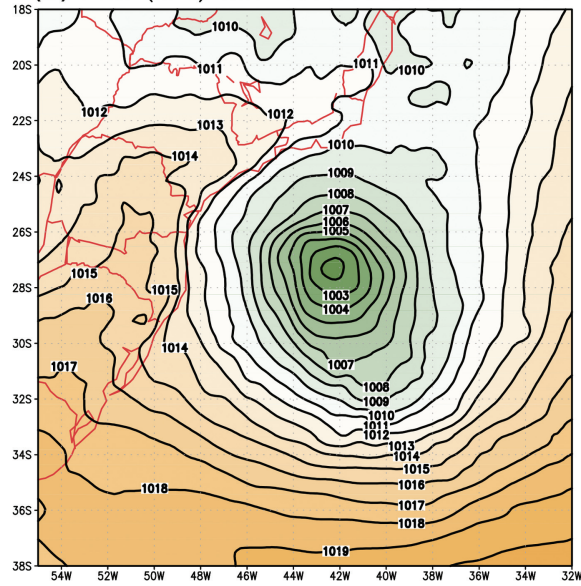

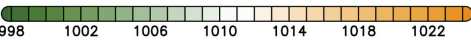

(d) PNM (hPa) MIN=1003.74 06Z23MAR2004

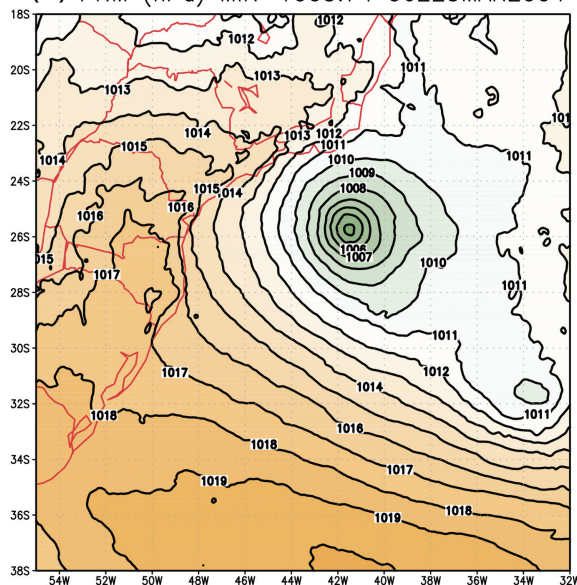

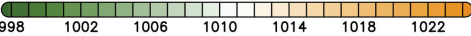

Figure 11. Sea level pressure (hPa) for the Hurricane Catarina on 23 March 2004. (a) Weather Research and Forecasting (WRF)-only high resolution. (b) Coupled ocean-atmosphere (COA) high resolution. (c) WRF-only very-high resolution. (d) COA very-high resolution.

they were $1000 \mathrm{hPa}(\mathrm{WRF})$ and $1003 \mathrm{hPa}(\mathrm{COA})$. Those values found for the 23 September simulations are in agreement with Mctaggart-Cowan et al. (2006), in which they estimated values ranging from 1002 to $990 \mathrm{hPa}$ during that day. However, analyzing the subsequent days of the simulations the resolution turns out to be crucial, because, in both experiments 
(a) PNM (hPa) MIN=1008.56 18Z27MAR2004
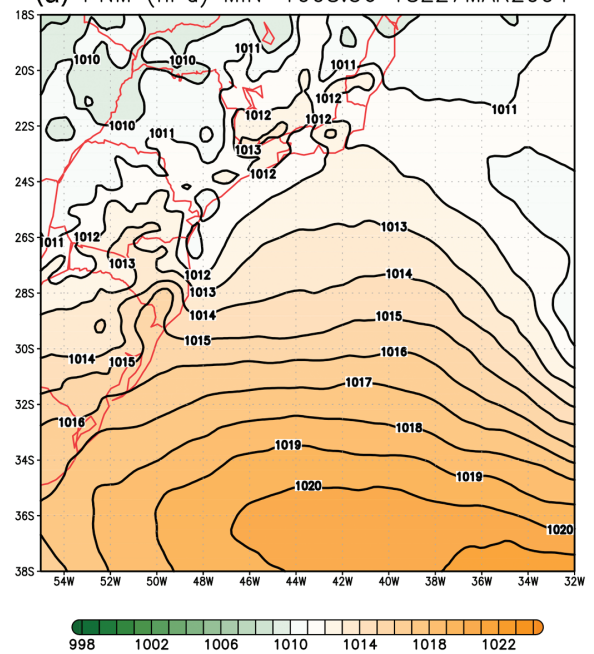

(c) PNM (hPa) MIN=1004.33 18Z27MAR2004

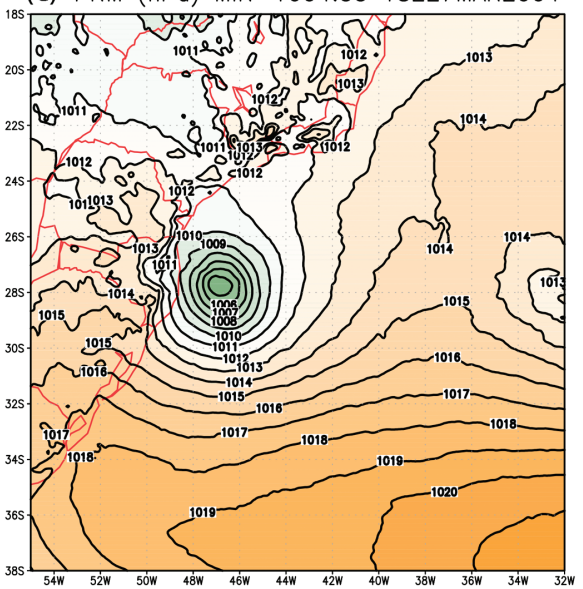

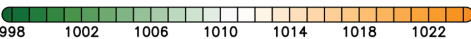

(b) PNM (hPa) MIN=1008.66 18Z27MAR2004

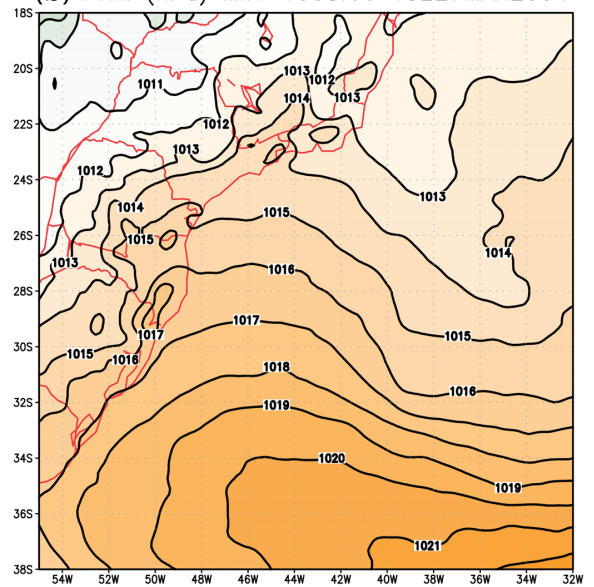

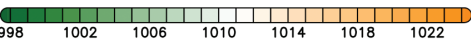

(d) PNM (hPa) MIN=1002.8 18Z27MAR2004

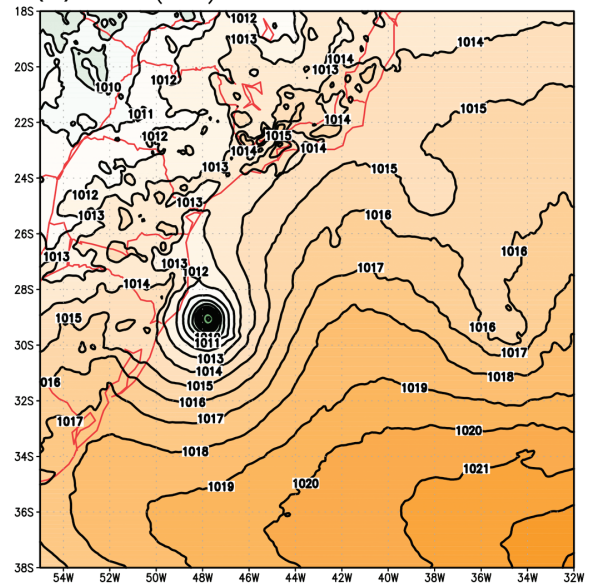

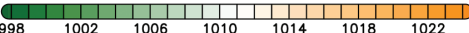

Figure 12. Sea level pressure (hPa) for the Hurricane Catarina on 27 March 2004. (a) Weather Research and Forecasting (WRF)-only high resolution. (b) Coupled ocean-atmosphere (COA) high resolution. (c) WRF-only very-high resolution. (d) COA very-high resolution.

using 20-km horizontal resolution, the cyclone intensity is decreased and it dissipates before making landfall on the continent (Figure 12, upper panels).

On the other hand, the simulations indicate that the phenomenon is relatively well represented in the 6-km case (Figure 12, lower panels). Nevertheless, the very-high resolution 
simulations for both WRF and COA do not lower the pressure center as much as the one estimated by Mctaggart-Cowan et al. (2006), being $1004 \mathrm{hPa}$ (WRF) and $1002 \mathrm{hPa}$ (COA), respectively, whereas the estimate was near $974 \mathrm{hPa}$.

The differences found in the simulations may have several causes, but, as discussed in Sections $2 \mathrm{c}$ and $3 \mathrm{a}$, the grid resolution may be playing a determinant role in calculating the surface fluxes by the atmospheric model bulk BL scheme, which is used in both WRF and COA experiments. The resulting fluxes found in the case of very-high resolution simulations better represent the smaller-scale features in the SST, whereas in the lower resolution simulations these features are smoothed (Figure 13). This smoothing occurs both when the oceanic model is used at lower resolution and when WRF-only is used with prescribed SST. Together with the conditional instability of the second kind (CISK) mechanism, the heat transferred from ocean to atmosphere in cyclones in their mature phase is the mechanism that maintains these phenomena (Rotunno and Emanuel 1987). In this way the cyclones are also maintained by the energy transferred from the ocean to atmosphere via sensible and mainly latent heat flows in tropical regions. This is the case seen in the very-high resolution experiments (Figure 13[c] and [d]) where the latent heat fluxes are still higher undeneath the hurricane, compared to high-resolution experiments where Catarina has vanished (Figure $13[\mathrm{a}]$ and [b]).

In addition to the resolution issue, the coupled simulation (COA), shown in Figure 14, can simulate the Hurricane Catarina eye closer to the place where it touched the continent, compared to the WRF-only experiment. This COA result is in very close agreement to the track estimation by McTaggart-Cowan et al. (2006). Thus the value of high-resolution grids for capturing dynamic processes in strongly coupled regimes is reinforced. This study adds to a growing body of literature documenting the superior skill that coupled (air-sea and air-sea-wave) models produce for hurricane prediction (e.g., Lee and Chen 2012; Chen and Curcic 2016).

\section{Medium-term (multiple weeks) applications}

\section{a. Impact of temporal frequency of exchange}

In the arena of medium-term (several-week horizon) forecasts, the demands of managing computational resources can outweigh the considerations that would enhance model fidelity. This is because, for medium-range simulations, the domains are necessarily larger. To that end, we examine the gains enabled by more-frequent coupling exchange between the air and sea in the context of medium-range projections.

A key target of medium-range prediction is the MJO (Zhang 2005). The MJO is the dominant form of intraseasonal variability in the tropical atmospheric system, characterized by large-scale, eastward-propagating, equatorially trapped, baroclinic oscillations in the tropical wind field at periods of 30-90 days (Madden and Julian 1971, 1994). MJO has predictability time scales of 10-30 days, which exceeds classical weather prediction timescales (e.g., Hendon et al. 2000; Waliser, Murtugudde, and Lucas 2003). Despite recent 
(a) LHF MAX=464.102 18Z27MAR2004
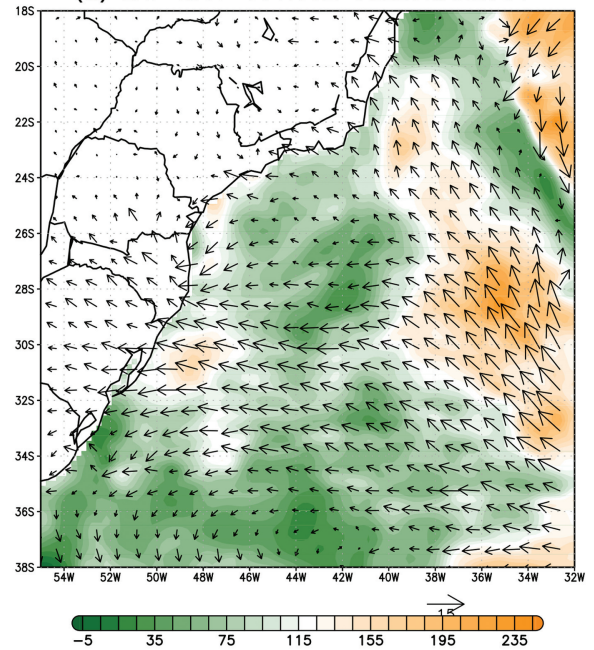

(c) LHF MAX=459.862 18Z27MAR2004
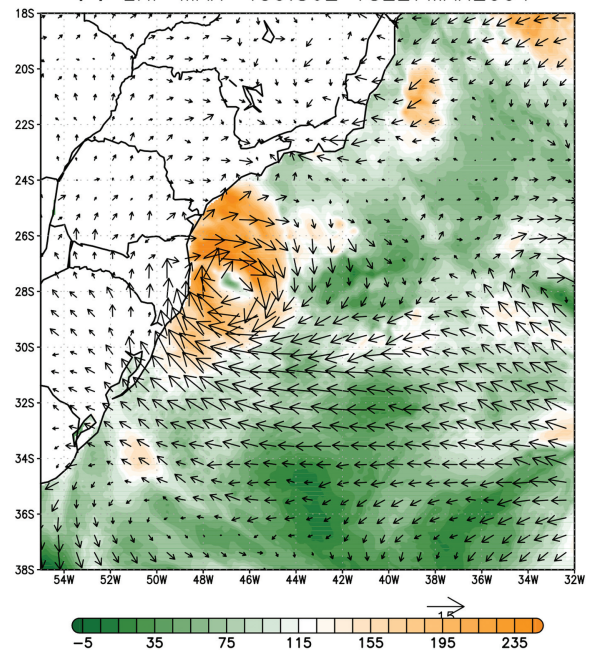

(b) LHF MAX $=458.805$ 18Z27MAR2004
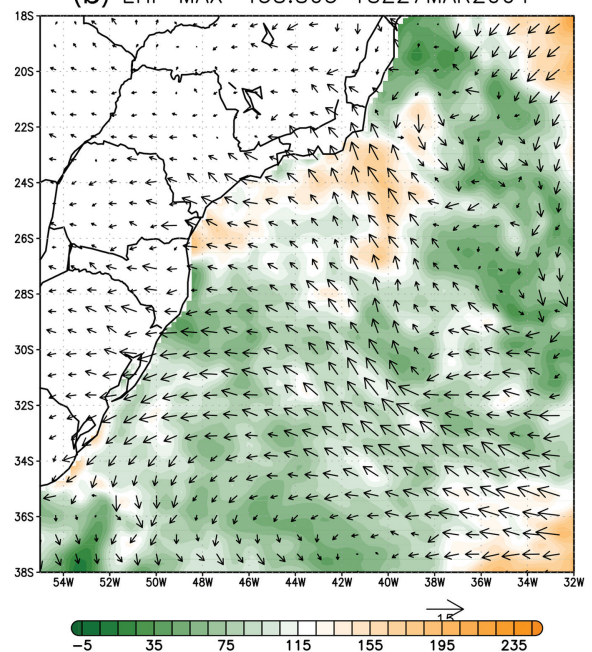

(d) LHF MAX $=469.018$ 18Z27MAR2004
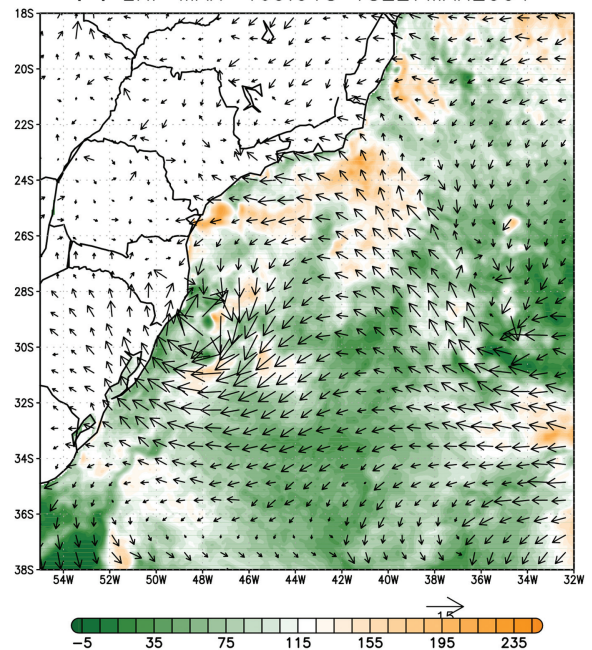

Figure 13. Latent heat flux $\left(\mathrm{W} / \mathrm{m}^{2}\right)$ and surface wind vectors $(\mathrm{m} / \mathrm{s})$ for the Hurricane Catarina on 27 March 2004. (a) Weather Research and Forecasting (WRF)-only high resolution. (b) Coupled ocean-atmosphere (COA) high resolution. (c) WRF-only very-high resolution. (d) COA very-high resolution.

advancements in MJO simulation and prediction in the realistic full-physics global climate models and numerical weather prediction models (e.g., Woolnough, Vitart, and Balmaseda 2007; Neale at al. 2008; Vitart and Molteni 2010; Subramanian et al. 2011), many aspects of the generation and maintenance of MJO remain elusive. These models lack many important 


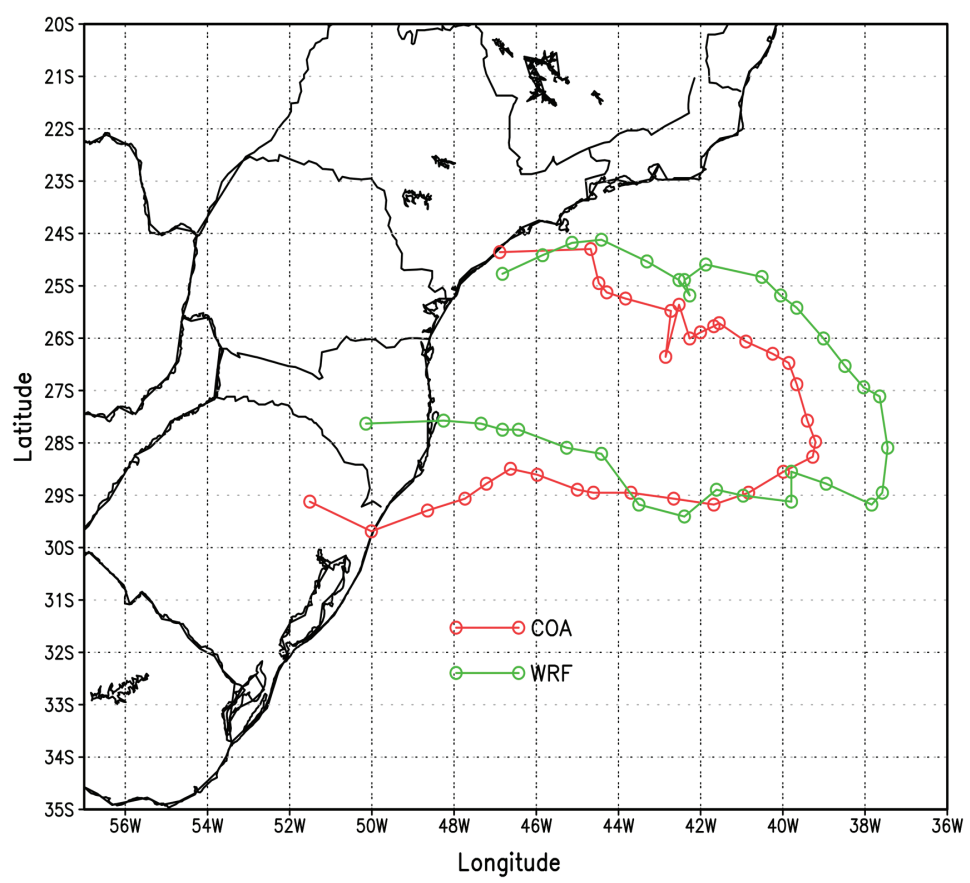

Figure 14. Estimated track for Hurricane Catarina extracted from the very-high resolution experiments using Weather Research and Forecasting (WRF)-only (green line) and fully coupled ocean and atmosphere (COA) (red line).

features of MJO because the multiscale interactions involving clouds and the oceans during initiation, growth, and propagation (Zhang 2005; Lin et al. 2006; Subramanian et al. 2011; Hung et al. 2013) are not well represented at coarse-grid resolutions.

Regional climate models, which are driven by boundary conditions from reanalysis products or free-running global climate models, could provide a unique opportunity to identify key processes affecting MJO, such as initiation and growth of the MJO and the role of air-sea interactions (Flatau et al. 1997). Upper-ocean processes determine mixed-layer heat content, stratification, and mixing, which alter the SST and the surface heat flux. The ensuing air-sea interactions are an important element for initiation and evolution of the MJO (Waliser, Lau, and Kim 1999; Zhang et al. 2006). The convection and the associated winds and incident solar radiation, in turn, generate intraseasonal variations in SST, feeding back to the intraseasonal variations in tropical atmospheric circulations associated with the MJO.

Regional coupled models, however, have been only recently used for MJO simulation. Shinoda et al. (2013), for example, used the COAMPS regional coupled model with multinested grids and data assimilation in the atmosphere to demonstrate that the upper ocean response to the westerly wind bursts associated with the March-April 2009 MJO event was 
sensitive to the horizontal resolution of the atmospheric model. The equatorial jet of up to $1 \mathrm{~m} / \mathrm{s}$ and the response in the upper ocean salinity and temperatures were significantly underestimated if the atmospheric resolution was reduced from $9 \mathrm{~km}$ to $27 \mathrm{~km}$. This study also demonstrates the pronounced diurnal SST warming during the suppressed phase of the MJO, but their impact on the MJO convection has not been evaluated. And utilizing a high-resolution two-way coupled model, Pullen et al. (2015) demonstrated the influence of $\mathrm{MJO}$ on the rainfall patterns in the mountains of the MC.

A recent study by Seo et al. (2014) adopted a high-resolution, tropical-channel version of the SCOAR model (WRF-ROMS) to evaluate the impact of preconvection SST warming associated with diurnal temperature variability in the upper ocean on the initiation and maintenance of the MJO. A tropical channel configuration provides several benefits for the study of the MJO. Without the east-west lateral boundary conditions, it allows the circumequatorial atmospheric disturbances to freely evolve (e.g., Ray et al. 2009, 2011) and interact with the ocean at high resolution. For this reason, a tropical channel model approach has been often used for the MJO simulations in the previous atmosphere-only regional models. For example, using the tropical-channel WRF model, Ray et al. (2009) demonstrated the critical role of time-varying lateral boundary conditions for initiation of MJO by allowing meridional advection of the westerly momentum from the extratropics.

Using a series of the tropical-channel SCOAR coupled model simulations employing varying coupling frequencies $(\mathrm{CF})$ from one-hour to daily, the impact of the preconvection SST on the MJO onset and intensification was quantified (Seo et al. 2014), as shown in Figure 15. The SCOAR in the tropical channel configuration represents reasonably well the observed eastward propagating precipitation anomalies associated with the observed MJO in November 2011 (Figure 15[a-d]). Although some predictability of the large-scale circulation associated with the MJO might have been provided by the initial and lateral boundary conditions, sensitivity experiments with different CFs (Figure 15[e]) show a clear sensitivity of the initiation and intensity of the MJO deep convection to the preconvection diurnal SST variability. The simulated precipitation amount associated with the MJO becomes much smaller when the model is coupled hourly to once daily (CF24). This is because the inclusion of resolved, interactively-generated diurnal SST variability nonlinearly rectifies and raises the time-mean SST and latent heat flux, preconditioning the local atmosphere for the deep convection. Diurnal SST variability also strengthens the diurnal moistening of the troposphere by collocating the diurnal peak in latent heat flux with that of SST. Both effects enhance the deep convection such that the total precipitation amount scales quasilinearly with preconvection diurnal SST amplitude and time-mean SST. However, the timing of peak convection is not strongly influenced by the diurnal SST variability, suggesting that it may be more sensitive to the initial and lateral boundary conditions. A complementary WRF-only simulation forced with the persistent initial SST (CF1PS), lacking the enhanced preconvection warming and moistening, produces a weaker and delayed convection. The fact that time-evolving SST with a diurnal cycle strongly influences the onset and intensity of the MJO deep convection suggests that improved representation of 
(a) TRMM precip

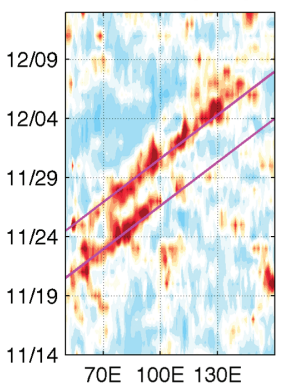

(b) CF1

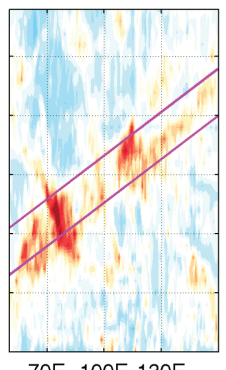

70E 100E 130E (c) CF24

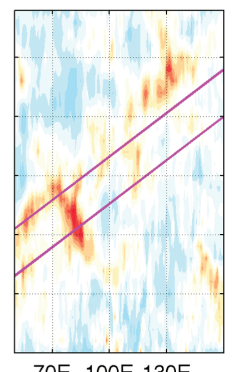

70E 100E 130E (d) CF1DM

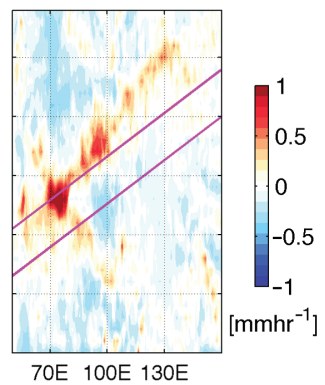

(e) Precip at $73-80.5^{\circ} \mathrm{E} 0.7^{\circ} \mathrm{S}-7^{\circ} \mathrm{N}$

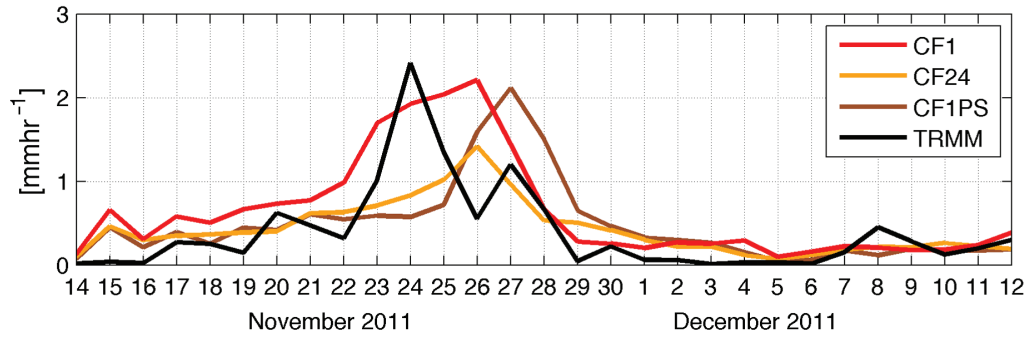

Figure 15. (a-e) Time-longitude diagrams of daily mean precipitation rate anomaly $\left(\mathrm{mmhr}^{-1}\right.$ ) averaged in $10^{\circ} \mathrm{S}-10^{\circ} \mathrm{N}$ from (a) Tropical Rainfall Measuring Mission (TRMM)-3B42, (b) 1-hourly coupled Scripps Coupled Ocean-Atmosphere Regional (SCOAR) model simulation (CF1), (c) daily coupled (CF24), and (d) Weather Research and Forecasting (WRF)-only simulation forced with daily mean SST obtained from CF1 (CF1DM). Two magenta diagonal lines, common to all the panels, denote the $8-\mathrm{ms}^{-1}$ phase lines derived from (a). (e) Time-series of daily mean total precipitation rates $\left(\mathrm{mmhr}^{-1}\right)$ averaged over the central Indian Ocean $\left(73-80.5^{\circ} \mathrm{E}\right.$ and $\left.0.7^{\circ} \mathrm{S}-7^{\circ} \mathrm{N}\right)$ for TRMM, CF1, CF24, and WRF only simulation forced with persistent initial sea surface temperature (SST) (CF1PS).

diurnal SST might help to produce more reliable forecasts of MJO onset and growth for enhancing medium-range predictability (DeMott, Klingaman, and Woolnough 2015).

\section{b. Role of ocean current feedback}

The energetic effects of surface currents on regional atmospheric forecasts for the medium-range are a manifestation of the momentum transfer between models (Section $3 b$ ). This aspect of coupling is frequently not fully appreciated as a factor in the formulation of the coupling interface. Therefore, we close with an explicit examination of the role of surface current feedback in the context of a coupled model.

Tropical instability waves (TIWs) occur in the eastern tropical Pacific and tropical Atlantic Oceans along the SST fronts associated with the equatorial cold tongues. These SST undulations are observed to have horizontal wavelengths of 1,000 to 2,000 km and periods of 20 to 30 days, propagating westward at approximately $0.5 \mathrm{~m} / \mathrm{s}$ (Qiao and Weisberg 1995). 
Observations and numerical studies have shown that TIWs are generated through instability of the equatorial current and frontal system (e.g., Philander 1976, 1978; Cox 1980; Hansen and Paul 1984; Yu, McCreary Jr., and Proehl 1995), and play a leading role in the upper ocean heat balance in the equatorial cold tongue (e.g., Jochum, Malanotte-Rizzoli, and Busalacchi 2004; Pezzi et al. 2004, Jochum and Murtugudde 2006; Menkes et al. 2006).

These intraseasonal undulations of SST fronts by TIWs induce surprisingly coherent responses in the surface and lower-tropospheric fields (e.g., Hashizume et al. 2002; Chelton et al. 2004; Pezzi et al. 2004). This covariability in ocean and atmospheric fields on intraseasonal time-scales driven by the ocean dynamics has been a topic of several regional coupled modeling studies, given their role in the upper ocean heat balance. The dynamics of coupling and impact on the large-scale fields by TIWs were examined in a series of SCOAR model simulations by Seo et al. (2006; 2008a) and Seo, Miller, and Roads (2007), and Seo and Xie (2011). They reveal that TIW-induced SST and surface current anomalies exert significant damping effect on the thermodynamic and energetics of the TIWs, influencing the propagation characteristics and the upper-ocean heat budget and the overlying atmospheric precipitation variations. Using the International Pacific Research Center (IPRC) regional coupled model (IROAM), a series of papers (Xie et al. 2007; de Szoeke et al. 2007; Small et al. 2009) also diagnose the effect of TIWs on the heat budget, SST, and eddy kinetic energy in the eastern equatorial Pacific. These studies demonstrate that including the feedback effect by TIWs on the atmosphere via SST and surface currents in the coupled model influences predictability aspects of both the atmospheric and ocean flows in this region. This effect will need to be included in regional forecast experiments to quantify their impact, especially in oceanic regions with strong eddy activities, such as in the California Current System (Seo et al. 2016; Renault et al. 2016b) and in western boundary current regions. In these regions, the current feedback may influence the low-level baroclinicity/storm track variability (e.g., Renault 2016a) and horizontal moisture transport (Seo 2017) in the atmosphere.

\section{Coupling challenges and advances}

\section{a. Coupled model initialization}

As limited area models, regional coupled systems require high-fidelity inputs to maximize forecast skill. When setting up a new region for a coupled modeling system, consideration must be given to the length of time required to spin up the individual components of the system. It is usually difficult for basin-scale and global general circulation models to reach an equilibrium state, as it can take hundreds of years. Typically, an ocean model is initialized with the present ocean state and is integrated forward until the circulation is consistent with the prescribed water mass structure. In other words, it adjusts geostrophically to its initial state. However, the ocean initial state is imperfect due to the sparseness of data at depth. In contrast, the atmospheric model, if run uncoupled, can typically be spun-up over a period of several days to resolve the synoptic features in the region. Similarly, a minimum period of a week is required to spin-up a regional wave model to adequately 
resolve swell propagating into the model domain from distant locations, depending on the geographic region of interest. When a regional coupled model system is initialized from a cold start, each component is initialized from a global atmosphere, ocean, and wave model. These same models are used to provide boundary conditions for the respective model. The ready availability of global fields and all requisite atmosphere and ocean observations is essential. These requirements make demands on the storage and computational systems at hand.

\section{b. Coupled model data assimilation}

Assimilation techniques are used to make modeled fields resemble atmospheric and oceanic circulation patterns observed (and measured) at a given time and place, and to make predictions of future oceanic and atmospheric circulations from the initial state. Data assimilation is also useful to understand previous patterns of variability. The above timespan to achieve the initial state assume that both the atmosphere and ocean models (both global and regional) are being run in a data assimilative mode.

There are two types of assimilation schemes: direct insertion and incremental insertion. In the first scheme, data is inserted whenever it becomes available (global models typically look back for observations at least 72 hours). This has some advantages for the ocean, where in situ data is sparse and doesn't necessarily arrive on a regular schedule. The second type, incremental insertion, uses new observations by introducing the data gradually over time in order to minimize the imbalances that result from mismatches between the model and the data. Often, a hindcast with data assimilation is performed before a forecast is generated in order to utilize observations to provide the best possible initial state for the pending forecast.

An important aspect to data assimilation is quality control of the observational data. Quality control checks of the data are performed to flag data outliers that fall outside of 1 to 3 standard deviations of the data, usually based on climatology.

In general, the update cycle chosen in a coupled system is based upon the available observational data for each medium, as well as the dynamics of the fluid involved. Because atmospheric observations are much greater in number than oceanic observations, a sixhourly-update cycle with data assimilation may be adequate to capture the initial state and provide an accurate forecast. On the other hand, the ocean model may require a 24-hour or once-per-day update cycle due to the limited number of observations.

Increasingly, consistent assimilation across the air-sea boundary is viewed as the most reliable means of generating a coherent initial and updated state. In particular, "stronglycoupled" data assimilation, in which observations within one realm affect the other constituents (with some type of constraints), may represent a significant path to enhanced predictability of coupled systems (e.g., Frolov et al. 2015; Lu et al. 2015).

Related advances include the incorporation of data assimilation into other coupled model components. For example, the COAMPS effort includes incorporation of a wave data assimilation method (Veeramony et al. 2014) under development. And COAMPS is being extended 
for Arctic domains by coupling to the Community Ice CodE (CICE) sea ice model for a relocatable COAMPS-CICE modeling system. ROMS is also being coupled to CICE using the Model Coupling Toolkit (MCT). This is an active area of research and will certainly be represented in more coupled models of the future.

As more components of the earth system are drawn into coupled model interfaces, attention needs to be given to the underlying assumptions of the individual models. For instance, many components were tuned to run in a standalone manner. These issues are exacerbated by data assimilation schemes (and hence motivated the need for coupled data assimilation), but these challenges exist even in the absence of assimilation. Such drivers necessitate a perpetual examination of the component model physics and associated underlying parameterizations to confirm that their validity is upheld in the context of fully two-way coupled modeling systems.

\section{Summary}

As the studies mentioned and presented herein attest, there is a growing body of research and operational experience with coupled models to draw on in advancing the field. This paper has overviewed multiple ways in which coupled forecasting improves the predictive skill of both retrospective and operational simulations.

Coupled air-sea modeling advances have relied on a variety of different field campaigns over the past decade to refine and validate coupled modeling systems. These include Coupled Boundary Layers Air-Sea Transfer (CBLAST, 2001-2003), Adriatic Circulation Experiment (ACE, 2002-2003), Ligurian Sea Air-Sea Interaction Experiment (LASIE07, 2007), VAMOS Ocean Cloud Atmosphere Land Study (VOCALS, 2008), Philippine Straits Dynamics Experiment (PhilEx, 2007-2009), CYNDY/DYNAMO (2011-2012), and AirSea Interaction Regional Initiative in the Northern Indian Ocean (ASIRI, 2014-2016).

The application of coupled models is progressing from documenting when, where, and how coupling matters to the holistic adoption of coupled systems in research and operations. The Earth System Prediction Capability (ESPC) is a U.S. initiative to coordinate multirealm coupled prediction across federal agencies (Carman et al. 2017) utilizing a common infrastructure and ESMF-based coupling (Theurich et al. 2016). A particularly compelling development is the unified model approach whereby a single modeling suite is employed targeting subseasonal to seasonal timescales that bridge weather and climate (National Research Council 2016). Yet challenges remain in seamless initialization, in the integration of the models tuned to individual realms, and in the further development of data assimilation, especially coupled data assimilation.

Short time-scale forecasting clearly benefits from including highly resolved physical processes that currently require regional coupled models to properly implement. Future increases in computational efficiency will allow ever more finely-resolved grids and ever more complicated representations of physical processes in these models, potentially extending to global grids. Carefully designed sensitivity studies and skill assessments, with 
accompanying observational verification, need to support these types of simulations and forecasts in order to verify that the mechanisms leading to skillful predictions are correctly represented in the models. Such studies and assessments will also facilitate emerging advances in data assimilation and their incorporation and validation in coupled air-sea prediction systems. These steps will also be carried out across more components of the earth system in emerging coupled model frameworks.

On the research to operations front, operational coupled systems continue to proliferate in international environmental prediction centers such as the European Center for Mediumrange Weather Forecasting (ECMWF), Japan Agency for Marine-Earth Science and Technology (JAMSTEC), the UK Met Office, and the Canadian Meteorological Center, among others (Smith, Roy, and Brasnett 2013; Fallmann et al. 2017). And increasingly, international projects are linking modeling and observational capabilities. An upcoming program, the Years of the Maritime Continent (2017-2019; http://www.jamstec.go.jp/ymc/), is an example of a multicountry initiative to model and measure coupled processes related to MJO propagation across the land region between the Indian and Pacific Oceans. It offers an unprecedented opportunity to examine configuration and parameterization choices and their associated outcomes across multiple coupled models. Further advances will be achieved in this way by exercising coupled models side-by-side in operational and field campaign settings.

\section{Appendix}

\section{a. COAMPS}

Coupled Ocean/Atmosphere Mesoscale Prediction System (COAMPS), developed by the Naval Research Laboratory is used to study and predict short-term (3 to 7 days) atmospheric and oceanic phenomena for research, operations, and real-time experiment support. The atmospheric component of COAMPS (Hodur 1997; Chen et al. 2003) is a nonhydrostatic, terrain-following, and cloud-resolving nested model that includes parameterizations for subgrid-scale mixing, cumulus cloud parameterizations, and moist physics. A tropical cyclone version of the model (COAMPS-TC) (Doyle et al. 2014) has been developed for the prediction of cyclone track, intensity, and structure.

The ocean component is the Navy Coastal Ocean Model (NCOM) developed by the Naval Research Laboratory for regional and coastal ocean modeling (Martin 2000; Barron 2006; Allard et al. 2014; Rowley et al. 2014) of ocean temperature, salinity, currents, and sea surface height. The vertical grid levels in NCOM consist of a set number of terrainfollowing sigma levels from the surface down to a user-specified depth, with z-levels below. The model has numerous mixing parameterizations, includes tides, and has an option to run with real-time river discharge. Wave forcing terms including the Stokes drift current, gradients of radiation stress, and enhancement of vertical mixing by Langmuir turbulence as described by Kantha and Clayson (2004) have been incorporated into NCOM.

The wave component options in COAMPS are the Simulating Waves Nearshore (SWAN) (Booij, Ris, and Holthuijsen 1999; SWAN Team 2010) and WAVEWATCH III (Tolman 
2009, Rogers, Dykes, and Wittmann 2014). SWAN is designed to run efficiently at high resolutions (e.g., $2 \mathrm{~km}$ or finer) through the utilization of implicit propagation methods. SWAN can be run in nested mode receiving boundary conditions from a global or regional WAVEWATCH III. Recent modifications to the SWAN wave model include a new windto-wave energy input and wave-breaking energy dissipation source functions (Babanin et al. 2010) and a new ocean surface drag formulation for high-wind conditions. The new features showed significant improvement in forecasting wave properties during tropical cyclone conditions. In COAMPS, WAVEWATCH III is typically run as a regional wave model while SWAN is used for nearshore domains.

The system is coupled using the Earth System Modeling Framework (ESMF) (Campbell et al. 2010; Chen et al. 2010) and supports air-ocean (Small et al. 2011), air-wave, oceanwave, and air-ocean-wave coupling. The wind-wave coupling consists of the atmospheric model providing wind forcing to the wave model and the wave model providing feedback via a sea-state-dependent Charnock parameter, which is used to compute the atmospheric momentum drag at the air-sea interface. The exchange of fields can be set by the user (e.g., hourly) but typically ranges between 5 and 10 minutes.

The atmospheric data assimilation system in COAMPS is the Navy Atmospheric Variational Data Assimilation System (NAVDAS); Daley and Barker 2000) that assimilates conventional observations such as surface, upper-air, and satellite data. NCOM assimilates real-time ocean observational data including remotely-sensed SST, sea surface height, in situ surface and subsurface observations of temperature and salinity, and measurements from ships, buoys, expendable thermographs, and floats using the Navy Coupled Ocean Data Assimilation (NCODA) system (Cummings 2005; Cummings and Smedstad 2013). NCODA is a fully three-dimensional, multivariate, optimal-interpolation (MVOI) ocean data assimilation system.

Each of the model components described receives initial/boundary (I/B) conditions from a global model. The NAVy Global Environmental Model (NAVGEM) (Hogan et al. 2014) is the Navy's operational global weather prediction model. NAVGEM combines a semi-Lagrangian/semi-implicit dynamical core with parameterizations for subgrid scale moist processes, convection, and radiation. This model is used for I/B conditions for the regional COAMPS atmospheric model. The U.S. Navy's operational global ocean nowcast/forecast system is comprised of the HYbrid Coordinate Ocean Model (HYCOM) and the Navy Coupled Ocean Data Assimilation (NCODA) system (Metzger et al. 2014).

Global HYCOM is used to provide I/B conditions for regional NCOM forecasts. WAVEWATCH III (Tolman 2009; Rogers, Dykes, and Wittmann 2014) is an operational global and regional model that is run at the NOAA National Center for Environmental Prediction (NCEP), the Fleet Numerical Meteorology and Oceanography Command, and the Naval Oceanographic Office. I/B conditions for this model are used for regional SWAN nowcast/forecast systems. 
The coupled system is typically spun-up for several weeks prior to generating forecast products. Typically, a 72-hr ocean forecast is generated twice daily (00, 12 UTC) in a coupled mode, with an atmospheric update cycle performed at 06 and 18 UTC.

Numerous validation studies for the system components have been performed for atmosphere-ocean (Allard et al., 2010; Jensen et al., 2015), ocean-wave (Allard et al., 2012), and atmosphere-wave coupling (Chen et al. 2014).

\section{b. COAWST}

The Coupled-Ocean-Atmosphere-Wave-Sediment-Transport System (COAWST) coupling is performed using the Model Coupling Toolkit. Some model specifications used in the Madeira archipelago simulation of Section 1 are described here, but data sources and model settings may vary depending on the region for which the open source code is configured. The initial and boundary atmospheric conditions are retrieved from the National Center for Environmental Prediction (NCEP) Final Analyses with a $1^{\circ}$ spatial resolution every 6 hours. WRF topography was retrieved from SRTM 3-second database, and, for land use type, the Corine Land Cover dataset is employed. For WRF, the following options were chosen: cloud microphysics use the WRF Single-Moment 6-class scheme (Hong, Noh, and Dudhia 2006), longwave radiation uses the Rapid Radiative Transfer Model (Mlawer and Clough 1997), shortwave radiation uses the Dudhia scheme (Dudhia 1989), the surface layer follows the Eta similarity scheme (Janjic 1996, 2002) and the planetary boundary layer follows the Mellor-Yamada-Janjic scheme (Janjic 1990, 1994).

Oceanic initial and boundary conditions are based on 5-day intervals of HYCOM (Hybrid Coordinate Ocean Model) + NCODA (Navy Coupled Ocean Data Assimilation) global $1 / 12^{\circ}$ analysis data. Raw bathymetry data were taken from the 30-second resolution, GEBCO (General Bathymetric Chart of the Oceans) bathymetry. In ROMS (Regional Ocean Modeling System), radiation and nudging boundary conditions are imposed for tracers (temperature and salinity) and baroclinic velocities (Marchesiello, McWilliams, and Shchepetkin 2001), a Flather condition for barotropic velocities (Flather 1976), and a Chapman boundary condition for free-surface height (Chapman 1985). Vertical turbulent mixing is parameterized with the Generic Length Scale (GLS) scheme, using a k-kl closure, where k is the turbulent kinetic energy and 1 is the length scale (Warner et al. 2005). The model state variables are staggered on an Arakawa C-grid.

Acknowledgments. JP acknowledges support from Office of Naval Research (ONR) grant N0001410-1-0300. RAA and TAS were supported through the 6.2 NRL Core Project "Coupled Ocean-Wave Prediction System" Program Element \#0602435N. HS acknowledges support from ONR (N0001415-1-2588), NSF (OCE-f 419235), and NOAA (NA15OAR4310176). AJM was supported by the NSF Earth System Modeling Program (OCE1419306) and the NOAA Climate Variability and Prediction Program (NA14OAR4310276). LPP is supported by CNPq's fellowships on scientific productivity (CNPq 304009/2016-4). This is also one of his contribution to the project Advanced Studies in Medium and High Latitudes Oceanography (CAPES 23038.004304/ 2014-28). JA and RC were 
financially supported by the Oceanic Observatory of Madeira Project (M1420-01-0145-FEDER000001-Observatório Oceânico da Madeira-OOM). This is NOAA GLERL Contribution Number 1854.

\section{REFERENCES}

Acevedo, O. C., L. P. Pezzi, R. B. Souza, V. Anabor, and G. Degrazia. 2010. Atmospheric boundary layer adjustment to the synoptic cycle at the Brazil-Malvinas Confluence South Atlantic Ocean. J. Geophys. Res., 115, D22107. doi: 10.1029/2009JD013785

Allard, R. A., T. J. Campbell, T. A. Smith, T. G. Jensen, S. Chen, J. A. Cummings, J. Doyle et al. 2010. Validation Test Report for the Coupled Ocean Atmosphere Mesoscale Prediction System (COAMPS) Version 5.0 NRL/MR/7322-10-9283. Stennis Space Center, MS: Oceanography Division, Naval Research Laboratory, 172 pp.

Allard, R., E. Rogers, P. Martin, T. Jensen, P. Chu, T. Campbell, J. Dykes et al. 2014. The US Navy coupled ocean-wave prediction system. Oceanography, 27(3), 92-103. doi: 10.5670/oceanog.2014.71

Allard, R. A., T. A. Smith, T. G. Jensen, P. Y Chu, E. Rogers, T. J. Campbell, U. M. Gravois et al. 2012. Validation Test Report for the Coupled Ocean/Atmosphere Mesoscale Prediction System (COAMPS) Version 5.0: Ocean/Wave Component Validation. NRL/MR/7320-12-9423, 91 pp.

Babanin, A. V., K. N. Tsagareli, I. R. Young, and D. J. Walker. 2010. Numerical investigation of spectral evolution of wind waves. Part II: Dissipation term and evolution tests. J. Phys. Oceanogr., 40, 667-683. doi: 10.1175/2009JPO4370.1

Bao, J. W., J. M. Wilczak, J. K. Choi, and L. H. Kantha. 2000. Numerical simulations of air-sea interaction under high wind conditions using a coupled model: A study of hurricane development. Mon. Weather Rev., 128(7), 2190-2210. doi: 10.1175/1520-0493(2000)128<2190:NSOASI > 2.0.CO;2

Barron, C. N., A. B. Kara, P. J. Martin, R. C. Rhodes, and L. F. Smedstad. 2006. Formulation, implementation and examination of vertical coordinate choices in the global Navy Coastal Ocean Model (NCOM). Ocean Model., 11, 347-375. doi: 10.1016/j.ocemod.2005.01.004

Barton, E. D., G. Basterretxea, P. Flament, E. G. Mitchelson-Jacob, B. Jones, J. Arístegui, and F. Herrera. 2000. Lee region of Gran Canaria. J. Geophys. Res.: Oceans, 105(C7), 17173-17193. doi: 10.1029/2000JC900010

Bender, M. A., I. Ginis, R. Tuleya, B. Thomas, and T. Marchok. 2007. The operational GFDL coupled hurricane-ocean prediction system and a summary of its performance. Mon. Weather Rev., 135, 3965-3989. doi: 10.1175/2007MWR2032.1

Bender, M. A. and I. Ginis. 2000. Real-case simulations of hurricane-ocean interaction using a highresolution coupled model: Effects on hurricane intensity. Mon. Weather Rev., 128, 917-946. doi: 10.1175/1520-0493(2000)128<0917:RCSOHO > 2.0.CO;2

Booij, N., R. C. Ris, and L. H. Holthuijsen. 1999. A third-generation wave model for coastal regions: Part 1. Model description and validation. J. Geophys. Res.: Oceans, 104(C4), 7649-7666. doi: 10.1029/98JC02622

Bourassa, M. A., D. G. Vincent, and W. L. Wood. 1999. A flux parameterization including the effects of capillary waves and sea state. J. Atmos. Sci., 56(9), 1123-1139. doi: 10.1175/15200469(1999)056<1123:AFPITE > 2.0.CO;2

Businger, J. A. and W. J. Shaw. 1984. The response of the marine boundary layer to mesoscale variation in sea-surface temperature. Dyn. Atmos. Oceans, 8, 267-281. doi: 10.1016/0377-0265(84)90012-5

Bye, J. A. T. 1986. Momentum exchange at the sea surface by wind stress and understress. Q. J. R. Met. Soc., 112, 501-510. doi: 10.1002/qj.49711247212 
Caldeira, R., X. Couvelard, R. Vieira, C. Lucas, I. Sala, and I. Vallès Casanova. 2016. Challenges of building an operational ocean forecasting system for small island regions: Regional to local. J. Oper. Oceanogr., 9(1), 1-12. doi: 10.1080/1755876X.2016.1205304

Caldeira, R. M. A., S. Groom, P. Miller, D. Pilgrim, and N. P. Nezlin. 2002. Sea-surface signatures of the island mass effect phenomena around Madeira Island, Northeast Atlantic. Remote Sens. Environ., 80(2), 336-360. doi: 10.1016/S0034-4257(01)00316-9

Caldeira, R. M. and R. Tomé. 2013. Wake response to an ocean-feedback mechanism: Madeira Island case study. Boundary Layer Meteorol., 148(2), 419-436. doi: 10.1007/s10546-013-9817-y

Camargo, R., E. Todesco, L. P. Pezzi, and R. B. Souza. 2013. Modulation mechanisms of marine atmospheric boundary layer at the Brazil-Malvinas Confluence region. J. Geophys, Res.: Atmos., 118, 6266-6280. doi: 10.1002/jgrd.50492

Campbell, T., R. Allard, R. Preller, L. Smedstad, A. Wallcraft, S. Chen, H. Jin et al. 2010. Integrated modeling of the battlespace environment. Comp. Sci. Eng., 12(5), 36-45. doi: 10.1109/ MCSE.2010.78

Carman, J. C., D. P. Eleuterio, T. C. Gallaudet, G. L. Geernaert, P. A. Harr, J. A. Kaye, D. H. McCarren et al. 2017. The National Earth System prediction capability: Coordinating the giant. Bull. Am. Meteorol. Soc., 98(2), 239-252. doi: 10.1175/BAMS-D-16-0002.1

Cayan, D. R. 1992. Latent and sensible heat-flux anomalies over the Northern Oceans: Driving the sea surface temperature. J. Phys. Oceanogr., 22, 859-881. doi: 10.1175/1520-0485(1992) $022<0859$ :LASHFA $>2.0$. CO; 2

Chapman, D. C. 1985. Numerical treatment of cross-shelf open boundaries in a barotropic coastal ocean model. J. Phys. Oceanogr., 15, 1060-1075. doi: 10.1175/1520-0485(1985) 015<1060:NTOCSO $>2.0 . \mathrm{CO} ; 2$

Chelton, D. B., P. Gaube, M. G. Schlax, J. J. Early, and R. M. Samelson. 2011. The influence of nonlinear mesoscale eddies on near-surface chlorophyll. Science, 334, 328-332. doi: 10.1126/science. 1208897

Chelton, D. B., M. Schlax, M. H. Freilich, and R. F. Milliff. 2004. Satellite measurements reveal persistent small-scale features in ocean winds. Science, 303, 978-983. doi: 10.1126/science.1091901

Chelton, D. B. and S.-P. Xie. 2010. Coupled ocean-atmosphere interaction at oceanic mesoscales. Oceanography, 23, 52-69. doi: 10.5670/oceanog.2010.05

Chen, S., T. Campbell, S. Gaberšek, R. Hodur, and P. J. Martin. 2010. Effect of two-way air-sea coupling in high and low wind speed regimes. Mon. Weather Rev., 138(9), 3579-3602. doi: 10.1175/2009MWR3119.1

Chen, S., J. Cummings, J. Doyle, R. H. Hodur, T. Holt, C. Liou, M. Liu et al. 2003. COAMPS Version 3 model description: General theory and equations. NRL/PU/7500-03-448, $145 \mathrm{pp}$.

Chen, S. S. and M. Curcic. 2016. Ocean surface waves in Hurricane Ike (2008) and Superstorm Sandy (2012): Coupled model predictions and observations. Ocean Model., 103, 161-176. doi: 10.1016/j.ocemod.2015.08.005

Chen, S., S. Gaberšek, J. D. Doyle, J. Cook, P. Y. Chu, R. A. Allard, P. A. Wittmann, and W. A. Nuss. 2014. Validation test report for the Coupled Ocean/Atmosphere Mesoscale Prediction System (COAMPS) Version 5.0: Air/Wave Component Validation. NRL/MR/7533-9526, 68 pp.

Chen, S. S., J. F. Price, W. Zhao, M. A. Donelan, and E. J. Walsh. 2007. The CBLAST- Hurricane program and the next-generation fully coupled atmosphere-wave-ocean models for hurricane research and prediction. Bull. Amer. Meteor. Soc., 88, 311-317. doi: 10.1175/BAMS-88-3-311

Cornillon, P. and K.-A. Park. 2001. Warm core ring velocity inferred from NSCAT. Geophys. Res. Lett., 28, 575-578. doi: 10.1029/2000GL011487 
Cox, M. D. 1980. Generation and propagation of 30-day waves in a numerical model of the Pacific. J. Phys. Oceanogr., 10, 1168-1186 doi: 10.1175/1520-0485(1980)010<1168:GAPODW> 2.0.CO;2

Cummings, J. A. 2005. Operational multivariate ocean data assimilation. Q. J. R. Meteorol. Soc., 131, 3583-3604. doi: 10.1256/qj.05.105

Cummings, J. A. and O. M. Smedstad. 2013. Variational data assimilation for the global ocean, in Data Assimilation for Atmospheric, Oceanic and Hydrologic Applications, vol. II. S. K. Park and L. Xu, eds. Berlin: Springer-Verlag, pp. 303-343

Daley, R. and E. Barker. 2000. The NAVDAS Source Book, Publication NRL/PJ/7530-01-441. NRL, Monterey, CA: Naval Research Laboratory, 163 pp.

Davis, C. A., and L. F. Bosart. 2004. The TT problem: Forecasting the tropical transition of cyclones. Bull. Amer. Meteor. Soc., 85(11), 1657-1662. doi: 10.1175/BAMS-85-11-1657

DeMott, C. A., N. P. Klingaman, and S. J. Woolnough. 2015. Atmosphere-ocean coupled processes in the Madden-Julian oscillation. Rev. Geophys., 53(4), 1099-1154. doi: 10.1002/2014RG000478 de Szoeke, S. P., S.-P. Xie, T. Miyama, K. J. Richards, and R. J. O. Small. 2007. What maintains the SST front north of the equatorial cold tongue? J. Clim., 20, 2500-2514. doi: 10.1175/JCLI4173.1

Doyle, J. D., R. M. Hodur, S. Chen, Y. Jin, J. R. Moskaitis, S. Wang, E. A. Hendricks et al. 2014. Tropical cyclone prediction using COAMPS-TC. Oceanography 27(3), 104-115. doi: 10.5670/ oceanog.2014.72

Doyle, J. D., Q. Jiang, Y. Chao, and J. Farrara. 2009. High-resolution real-time modeling of the marine atmospheric boundary layer in support of the AOSN-II field campaign. Deep Sea Res. Part II Top. Stud. Oceanogr., 56(3), 87-99. doi: 10.1016/j.dsr2.2008.08.009

Dudhia, J. 1989. Numerical study of convection observed during the winter monsoon experiment using a mesoscale two-dimensional model. J. Atmos. Sci., 46, 3077-3107. doi: 10.1175/15200469(1989)046<3077:NSOCOD>2.0.CO;2

Eden, C. and H. Dietze. 2009. Effects of mesoscale eddy/wind interactions on biological new production and eddy kinetic energy, J. Geophys. Res., 114, C05023. doi: 10.1029/2008JC005129

Fairall, C. W., E. F. Bradley, J. E. Hare, A. A. Grachev, and J. B. Edson. 2003. Bulk parameterization of air-sea fluxes: Updates and verification for the COARE algorithm. J. Clim., 16, 571-591 doi: 10.1175/1520-0442(2003)016<0571:BPOASF $>2.0$. CO;2

Fairall, C. W., E. F. Bradley, D. P. Rogers, J. B. Edson, and G. S. Young. 1996. Bulk parameterization of air-sea fluxes for tropical ocean-global atmosphere Coupled-Ocean Atmosphere Response Experiment. J. Geophys. Res., 101, 3747-3764. doi: 10.1029/95JC03205

Fairall, C. W., J. D. Kepert, and G. J. Holland. 1994. The effect of sea spray on surface energy transports over the ocean. Global Atmos. Ocean Syst., 2(2-3), 121-142.

Fallmann, J., H. Lewis, J. Castillo, A. Arnold, and S. Ramsdale. 2017. Impact of sea surface temperature on stratiform cloud formation over the North Sea. Geophys. Res. Lett., 44(9) 4296-4303. doi: 10.1002/2017GL073105

Flather, R. A. 1976. A tidal model of the north-west European continental shelf. Memoires de la Societe Royale des Sciences de Liege, 6(10), 141-164.

Flatau, M., P. J. Flatau, P. Phoebus, and P. P. Niiler. 1997. The feedback between equatorial convection and local radiative and evaporative processes: The implication for intraseasonal oscillations. J. Atmos. Sci., 54, 2373-2386. doi: 10.1175/1520-0469(1997)054<2373:TFBECA > 2.0.CO;2

Frolov, D. S., C. H. Bishop, D. T. Holt, D. J. Cummings, and D. D. Kuhl. 2015. Facilitating stronglycoupled ocean-atmosphere data assimilation with an interface solver. Mon. Weather Rev., 144, 3-20. doi: 10.1175/MWR-D-15-0041.1 
Gaube, P., D. B. Chelton, R. M. Samelson, M. G. Schlax, and L. W. O'Neill. 2015. Satellite observations of mesoscale eddy-induced Ekman pumping. J. Phys. Oceanogr., 45, 104-132. doi: 10.1175/JPO-D-14-0032.1

Gaube, P., D. J. McGillicuddy, Jr., D. B. Chelton, M. J. Behrenfeld, and P. G. Strutton. 2014. Regional variations in the influence of mesoscale eddies on near-surface chlorophyll. J. Geophys. Res.: Oceans, 119, 8195-8220. doi: 10.1002/2014JC010111

Grubišić, V., J. Sachsperger, and R. M. Caldeira. 2015. Atmospheric wake of Madeira: First aerial observations and numerical simulations. J. Atmos. Sci., 72(12), 4755. doi: 10.1175/JAS-D-140251.1

Hafner, J. and S. P. Xie. 2003. Far-field simulation of the Hawaiian wake: Sea surface temperature and orographic effects. J. Atmos. Sci., 60(24), 3021-3032. doi: 10.1175/15200469(2003)060<3021:FSOTHW>2.0.CO;2

Hansen, D. and C. A. Paul. 1984. Genesis and effects of long waves in the equatorial Pacific. J. Geophys. Res., 89, 10431-10440. doi: 10.1029/JC089iC06p10431

Hashizume, H., S.-P. Xie, M. Fujiwara, M. Shiotani, T. Watanabe, Y. Tanimoto, W. T. Liu, and K. Takeuchi. 2002. Direct observations of atmospheric boundary layer response to SST variations associated with tropical instability waves over the eastern equatorial Pacific. J. Clim., 15, 33793393 doi: 10.1175/1520-0442(2002)015<3379:DOOABL > 2.0.CO;2

Hayes, S. P., M. J. McPhaden, and J. M. Wallace. 1989. The influence of sea surface temperature on surface wind in the eastern equatorial Pacific: Weekly to monthly variability. J. Clim., 2, 1500-1506 doi: 10.1175/1520-0442(1989)002<1500:TIOSST>2.0.CO;2

Hendon, H. H., B. Liebmann, M. Newman, J. D. Glick, and J. Schemm. 2000. Medium range forecast errors associated with active episodes of the Madden-Julian oscillation. Mon. Weather Rev., 128, 69-86. doi: 10.1175/1520-0493(2000)128<0069:MRFEAW > 2.0.CO;2

Hodur, R. M. 1997. The Naval Research Laboratory's Coupled Ocean/Atmospheric Mesoscale Prediction System (COAMPS). Mon. Weather Rev., 125, 414-430. doi: 10.1175/15200493(1997)125<1414:TNRLSC > 2.0.CO;2

Hodur, R., J. Pullen, J. Cummings, X. Hong, J. D. Doyle, P. Martin, and M. Rennick. 2002. The Coupled Ocean/Atmosphere Mesoscale Prediction System (COAMPS). Oceanography, 15(1), 8898. doi: 10.5670/oceanog.2002.39

Hogan, T. F., M. Liu, J. A. Ridout, M. S. Peng, T. R. Whitcomb, B.C. Ruston, C.A. Reynolds et al. 2014. The Navy global environmental model. Oceanography, 27(3), 116-125. doi: 10.5670/oceanog.2014.73

Hong, S.-Y., Y. Noh, and J. Dudhia. 2006. A new vertical diffusion package with explicit treatment of entrainment processes. Mon. Weather Rev., 134, 2318-2341. doi: 10.1175/MWR3199.1

Hong, S.-Y and H.-L. Pan. 1996. Nonlocal boundary layer vertical diffusion in a mediumrange forecast model. Mon. Weather Rev., 124, 1215-1238. doi: 10.1175/1520-0493(1996) 124<2322:NBLVDI>2.0.CO;2

Houze, R. A. 1997. Stratiform precipitation in regions of convection: A meteorological paradox? Bull. Amer. Meteor. Soc., 78, 2179-2196. doi: 10.1175/1520-0477(1997)078 <2179:SPIROC > 2.0.CO;2

Houze, R. A., S. G. Geotis, F. D. Marks, Jr., and A. K. West. 1981. Winter monsoon convection in the vicinity of North Borneo. Part I: Structure and time variation of the clouds and precipitation. Mon. Weather Rev., 109, 1595-1614. doi: 10.1175/1520-0493(1981)109<1595:WMCITV>2.0.CO;2

Howden, S. D. and R. Murtugudde. 2001. Effects of river inputs into the Bay of Bengal. J. Geophys. Res., 106(C9), 19825-19843. doi: 10.1029/2000JC000656 
Hu, C., E. T. Montgomery, R. W, Schmitt, and F.E. Muller-Karger. 2004. The dispersal of the Amazon and Orinoco River water in the tropical Atlantic and Caribbean Sea: Observation from space and S-PALACE floats. Deep Sea Res. Part II Top. Stud. Oceanogr., 51(10), 1151-1171. doi: 10.1016/j.dsr2.2004.04.001

Hung, M.-P., J.-L. Lin, W. Wang, D. Kim, T. Shinoda, and S. J. Weaver. 2013. MJO and convectively coupled equatorial waves simulated by CMIP5 climate models. J. Clim., 26, 6185-6214. doi: 10.1175/JCLI-D-12-00541.1

Hunke, E. C. and M. M. Holland. 2007. Global atmospheric forcing data for Arctic ice-ocean modeling, J. Geophys. Res., 112, C04S14. doi: 10.1029/2006JC003640

Jacob, R., J. Larson, and E. Ong. 2005. MxN communication and parallel interpolation in CCSM3 using the model coupling toolkit. Int. J. High Perform. Comput. Appl., 19(3), 293-307. doi: $10.1177 / 1094342005056116$

Janjic, Z. I. 1990. The step-mountain coordinate: Physical package. Mon. Weather Rev., 118(7), 1429-1443. doi: 10.1175/1520-0493(1990)118<1429:TSMCPP $>2.0 . C O ; 2$

Janjic, Z. I. 1994. The step-mountain eta coordinate model: Further developments of the convection, viscous sublayer, and turbulence closure schemes. Mon. Weather Rev., 122, 927-945, doi: 10.1175/1520-0493(1994)122<0927:TSMECM>2.0.CO;2

Janjic, Z. I. 1996. The surface layer in the NCEP Eta model, in Proceedings of the 11th Conference on Numerical Weather Prediction, Norfolk, VA, 19-23 August 1996; Boston: American Meteorological Society, pp. 354-355.

Janjic, Z. I. 2002. Nonsingular implementation of the Mellor-Yamada level 2.5 scheme in the NCEP Meso Model. NCEP Office Note 437. Camp Springs, MD: National Centers for Environmental Prediction, $61 \mathrm{pp}$.

Jensen, T. G., T. Shinoda, S. Chen, and M. Flatau. 2015. Ocean response to CINDY/DYNAMO MJOs in air-sea-coupled COAMPS. J. Meteor. Soc. Japan, 93A, 157-178, doi: 10.2151/jmsj.2015-049

Jochum, M., P. Malanotte-Rizzoli, and A. Busalacchi. 2004. Tropical instability waves in the Atlantic Ocean. Ocean. Model., 7, 145-163. doi: 10.1016/S1463-5003(03)00042-8

Jochum, M. and R. Murtugudde. 2006. Temperature advection by tropical instability waves. J. Phys. Oceanogr., 36, 592-605. doi: 10.1175/JPO2870.1

Kantha, L. H. and C. A. Clayson. 2004. On the effect of surface gravity waves on mixing in the oceanic mixed layer. Ocean Model., 6, 101-124. doi: 10.1016/S1463-5003(02)00062-8

Kelly, K. A., S. Dickinson, M. J. McPhaden, and G. C. Johnson. 2001. Ocean currents evident in satellite wind data. Geophys. Res. Lett., 28, 2469-2472. doi: 10.1029/2000GL012610

Kilpatrick, T., S.-P. Xie, and T. Nasuno. 2017. Diurnal convection-wind coupling in the Bay of Bengal. J. Geophys. Res.: Atmos., Submitted. doi: 10.1002/2017JD027271

Kuzmić, M., I. Janeković, J. W. Book, P. J. Martin, and J. D. Doyle. 2006. Modeling the northern Adriatic doublegyre response to intense Bora wind: A revisit. Journal of Geophysical Research: Oceans, 111(C3).

Larson, J., R. Jacob, and E. Ong. 2005. The Model Coupling Toolkit: A new Fortran90 toolkit for building multiphysics parallel coupled models. Int. J. High Perform. Comput. Appl., 19(3), 277292. doi: $10.1177 / 1094342005056115$

Lee, C. Y. and S. S. Chen. 2012. Symmetric and asymmetric structures of hurricane boundary layer in coupled atmosphere-wave-ocean models and observations. J. Atmos. Sci., 69(12), 3576-3594. doi: $10.1175 /$ JAS-D-12-046.1 
Lin, J.-L., G. N. Kiladis, B. E. Mapes, K. M. Weickmann, K. R. Sperber, W. Lin, M. C. Wheeler et al. 2006. Tropical intraseasonal variability in 14 IPCC AR4 climate models. Part I: Convective signals. J. Clim., 19, 2665-2690. doi: 10.1175/JCLI3735.1

Liu, W. T, K. B. Katsaros, and J. A. Businger, 1979. Bulk parameterization of the air-sea exchange of heat and water vapor including the molecular constraints at the interface. J. Atmos. Sci., 36, 1722-1735.

Love, B. S., A. J. Matthews, and G. M. S. Lister. 2011. The diurnal cycle of precipitation over the maritime continent in a high-resolution atmospheric model. Q. J. R. Meteorol. Soc., 137, 934-947. doi: 10.1002/qj.809

Lu, F., Z. Liu, S. Zhang, and Y. Liu. 2015. Strongly Coupled Data Assimilation Using Leading Averaged Coupled Covariance (LACC). Part I: Simple model study. Mon. Weather Rev., 143, 3823-3837. doi: 10.1175/MWR-D-14-00322.1

Madden, R. A. and P. R. Julian. 1971. Detection of a 40-50 day oscillation in the zonal wind in the tropical Pacific. J. Atmos. Sci., 28(5), 702-708. doi: 10.1175/1520-0469(1971)028<0702: DOADOI $>2.0 . \mathrm{CO} ; 2$

Madden, R. A. and P. R. Julian. 1994. Observations of the 40-50-day tropical oscillationA review. Mon. Weather Rev., 122(5), 814-837. doi: 10.1175/1520-0493(1994)122<0814: OOTDTO $>2.0 . \mathrm{CO} ; 2$

Mapes, B. E., T. T. Warner, and M. Xu. 2003. Diurnal patterns of rainfall in northwestern South America. Part III: Diurnal gravity waves and nocturnal convection offshore. Mon. Weather Rev., 131, 830-844. doi: 10.1175/1520-0493(2003)131<0830:DPORIN > 2.0.CO;2

Marchesiello, P., J. C. McWilliams, and A. Shchepetkin. 2001. Open boundary conditions for longterm integration of regional oceanic models. Ocean Model., 3(1), 1-20. doi: 10.1016/S14635003(00)00013-5

Martin, P. J., 2000. A Description of the Navy Coastal Ocean Model Version 1.0. NRL Report NRL/FR/7322-00-9962. Stennis Space Center, MS: Oceanography Division, Naval Research Laboratory, $42 \mathrm{pp}$.

McTaggart-Cowan, R., L. F. Bosart, C. A. Davis, E. H. Atallah, J. R. Gyakum, and K. A. Emanuel. 2006. Analyses of Hurricane Catarina. Mon. Weather Rev., 134, 3029-3053. doi: 10.1175/ MWR3330.1

Menkes, C. E., J. G. Vialard, S. C. Kennan, J. P. Boulanger, and G. V. Madec. 2006. A modeling of the impact of tropical instability waves on the heat budget of the eastern equatorial Pacific. J. Phys. Oceanogr., 36, 847-865. doi: 10.1175/JPO2904.1

Metzger, E. J., O. M. Smedstad, P. G. Thoppil, H. E. Hurlburt, J. A. Cummings, A. J. Wallcraft, L. Zamudio et al. 2014. US Navy operational global ocean and Arctic ice prediction systems. Oceanography, 27(3), 32-43. doi: 10.5670/oceanog.2014.66

Mlawer, E. J. and S. A. Clough. 1997. On the extension of rapid radiative transfer model to the shortwave region, in Proceedings of the 6th Atmospheric Radiation Measurement (ARM) Science Team Meeting, US Department of Energy, CONF-9603149. Washington, DC: US Department of Energy.

Mori S., J. I. Hamada, Y. I. Tauhid, M. D. Yamanaka, N. Okamato, F. Murata, N. Sakurai et al. 2004. Diurnal land-sea rainfall peak migration over Sumatera Island, Indonesian Maritime Continent, observed by TRMM satellite and intensive rawinsonde soundings. Mon. Weather Rev., 132, 20212039. doi: 10.1175/1520-0493(2004)132<2021:DLRPMO>2.0.CO;2

Mujumdar, M., K. Salunke, S. A. Rao, M. Ravichandran, and B. N. Goswami. 2011. Diurnal cycle induced amplification of sea surface temperature intraseasonal oscillations over the Bay 
of Bengal in summer monsoon season. IEEE Trans. Geosci. Remote Sens., 8(2), 206-210. doi: 10.1109/LGRS.2010.2060183

National Research Council. 2016. Developing a U.S. research agenda to advance subseasonal to seasonal forecasting. Washington, DC: National Academies Press, 372 pp.

Neale, R. B., J. H. Richter, and M. Jochum. 2008. The impact of convection on ENSO: From a delayed oscillator to a series of events. J. Clim., 21, 5904-5924. doi: 10.1175/2008JCLI2244.1

Pacanowski, R. C. 1987. Effect of equatorial currents on surface stress. J. Phys. Oceanogr., 17(6), 833-838. doi: 10.1175/1520-0485(1987)017<0833:EOECOS > 2.0.CO;2

Park, K.-A., P. Cornillon, and D. L. Codiga. 2006. Modification of surface winds near ocean fronts: Effects of Gulf Stream rings on scatterometer (QuikSCAT, NSCAT) wind observations. J. Geophys. Res., 111, C03021. doi: 10.1029/2005JC003016

Peatman, S. C., A. J. Matthews, and D. P. Stevens. 2014. Propagation of the Madden-Julian Oscillation through the Maritime Continent and scale interaction with the diurnal cycle of precipitation. Q. J. R. Meteorol. Soc., 140, 814-825. doi: 10.1002/qj.2161

Perlin, N., E. D. Skyllingstad, R. M., Samelson, and P. L. Barbour. 2007. Numerical simulation of air-sea coupling during coastal upwelling. J. Phys. Oceanogr., 37(8), 2081-2093. doi: 10.1175/JPO3104.1

Pezzi, L. P., R. B. de Souza, O. Acevedo, I. Wainer, M. M. Mata, C. A. Garcia, and R. de Camargo. 2009. Multiyear measurements of the oceanic and atmospheric boundary layers at the Brazil-Malvinas confluence region. J. Geophys. Res.: Atmos., 114(D19), D19103. doi: 10.1029/2008JD011379

Pezzi, L. P., R. B. Souza, M. S. Dourado, C. A. E. Garcia, M. M. Mata, and M. A. F. Silva-Dias. 2005. Ocean-atmosphere in situ observations at the Brazil-Malvinas confluence region. Geophys. Res. Lett., 32, L22603. doi: 10.1029/2005GL023866

Pezzi, L. P., J. Vialard, K. J. Richards, C. Menkes, and D. Anderson. 2004. Influence of ocean atmosphere coupling on the properties of tropical instability waves. Geophys. Res. Lett., 31, L16306. doi: 10.1029/2004GL019995

Philander, S. G. H. 1976. Instabilities of zonal equatorial currents. J. Geophys. Res., 81, 3725-3735. doi: 10.1029/JC081i021p03725

Philander, S. G. H. 1978. Instabilities of zonal equatorial currents, 2. J. Geophys. Res., 83, 3679-3682 doi: 10.1029/JC083iC07p03679

Pullen, J., R. Caldeira, J. D. Doyle, P. May, and R. Tomé. 2017. Modeling the air-sea feedback system of Madeira Island. J. Adv. Model. Earth. Syst., in revision. doi: 10.1002/2016MS000861

Pullen, J., J.D. Doyle, T. Haack, C. Dorman, R. Signell, and C. M. Lee. 2007. Bora event variability and the role of air-sea feedback. J. Geophys. Res., 112, C03S18. doi: 10.1029/2006JC003726

Pullen, J., J. D. Doyle, and R.P. Signell. 2006. Two-way air-sea coupling: A study of the Adriatic. Mon. Weather Rev., 134(5), 1465-1483. doi: 10.1175/MWR3137.1

Pullen, J. A., L. Gordon, J. Sprintall, C. M. Lee, M. A. Alford, J. D. Doyle, and P. W. May. 2011. Atmospheric and oceanic processes in the vicinity of an island strait. Oceanography, 24(1), 112121. doi: 10.5670/oceanog.2011.08

Qiao, L. and R. H. Weisberg. 1995. Tropical instability wave kinematics: Observations from the Tropical Instability Wave Experiment. J. Phys. Oceanogr., 100, 8677-8693 doi: 10.1029/95JC00305

Ray, P., C. Zhang, J. Dudhia, and S. S. Chen. 2009. A numerical case study on the initiation of the Madden-Julian oscillation. J. Atmos. Sci., 66, 310-331. doi: 10.1175/2008JAS2701.1

Ray, P., C. Zhang, M. W. Moncrieff, J. Dudhia, J. M. Caron, L. R. Leung, and C. Bruyere. 2011. Role of the atmospheric mean state on the initiation of the Madden-Julian Oscillation in a tropical channel model. Clim. Dyn., 36, 161-184. doi: 10.1007/s00382-010-0859-2 
Renault, L., M. J. Molemaker, J. Gula, S. Masson, and J. C. McWilliams. 2016a. Control and stabilization of the Gulf Stream by oceanic current interaction with the atmosphere. J. Clim., 46, 3439-3453. doi: 10.1175/JPO-D-16-0115.1

Renault, L., M. J. Molemaker, J. C. McWilliams, A. F. Shchepetkin, F. Lemaré, D. Chelton, S. Illig, and A. Hall. 2016b. Modulation of wind work by oceanic current interaction with the atmosphere. J. Phys. Oceanogr., 46, 1685-1704. doi: 10.1175/JPO-D-15-0232.1

Ricchi, A., M. M. Miglietta, P. P. Falco, A. Benetazzo, D. Bonaldo, A. Bergamasco, M. Sclavo, and S. Carniel. 2016. On the use of a coupled ocean-atmosphere-wave model during an extreme cold air outbreak over the Adriatic Sea. Atmos. Res. 172, 48-65. doi: 10.1016/j.atmosres.2015.12.023

Rogers, W. E., J. D. Dykes, and P. A. Wittmann. 2014. US Navy global and regional wave modeling. Oceanography, 27(3), 56-67. doi: 10.5670/oceanog.2014.68

Rotunno, R. and K. Emanuel. 1987. An air-sea interaction theory for tropical cyclones. Part II: Evolutionary study using a nonhydrostatic axisymmetric numerical model. J. Atmos. Sci., 44, 542-561. doi: 10.1175/1520-0469(1987)044 <0542:AAITFT > 2.0.CO;2

Rowley, C. and A. Mask. 2014. Regional and Coastal Prediction with the Relocatable Ocean Nowcast/Forecast System. Oceanography, 27(3), 44-55. JSTOR, JSTOR, www.jstor.org/stable/24862188

Saleh, F., V. Ramaswamy, N. Georgas, A. F. Blumberg, and J. Pullen. 2016. A retrospective streamflow ensemble forecast for an extreme hydrologic event: a case study of Hurricane Irene and on the Hudson River basin. Hydrology and Earth System Sciences, 20(7), 2649-2667.

Sangrà, P., M. Auladell, A. Marrero-Díaz, J. L. Pelegrí, E. Fraile-Nuez, A. Rodríguez-Santana, J. M. Martín et al. 2007: On the nature of oceanic eddies shed by the Island of Gran Canaria. Deep Sea Res. Part II Top. Stud. Oceanogr., 54(5), 687-709. doi: 10.1016/j.dsr.2007.02.004

Seo, H. 2017. Distinct influence of air-sea interactions mediated by mesoscale sea surface temperature and surface current in the Arabian Sea. J. Clim., 30, 8061-8080. doi: 10.1175/JCLI-D-16-0834.1

Seo, H., M. Jochum, R. Murtugudde, and A. J. Miller. 2006. Effect of ocean mesoscale variability on the mean state of tropical Atlantic climate. Geoyphs. Res. Lett., 33, L09606. doi: 10.1029/2005GL025651

Seo, H., M. Jochum, R. Murtugudde, A. J. Miller, and J. O. Roads. 2007. Feedback of tropical instability wave-induced atmospheric variability onto the ocean. J. Clim., 20, 5842-5855. doi: 10.1175/JCLI4330.1

Seo, H., M. Jochum, R. Murtugudde, A. J. Miller, and J. O. Roads. 2008a. Precipitation from African easterly waves in a coupled model of the tropical Atlantic. J. Clim., 21, 1417-1431. doi: 10.1175/2007JCLI1906.1

Seo, H., A. J. Miller, and J. R. Norris. 2016. Eddy-wind interaction in the California current system: Dynamics and impacts. J. Phys. Oceanogr., 46, 439-459. doi: 10.1175/JPO-D-15-0086.1

Seo, H., A. J. Miller, and J. O. Roads. 2007. The Scripps Coupled Ocean-Atmosphere Regional (SCOAR) model, with applications in the eastern Pacific sector. J. Clim., 20, 381-402. doi: 10.1175/JCLI4016.1

Seo, H., R. Murtugudde, M. Jochum, and A. J. Miller. 2008b. Modeling of mesoscale coupled oceanatmosphere interaction and its feedback to ocean in the western Arabian Sea. Ocean Model., 25, 120-131. doi: 10.1016/j.ocemod.2008.07.003

Seo, H., A. C. Subramanian, A. J. Miller, and N. R. Cavanaugh. 2014. Coupled impacts of the diurnal cycle of sea surface temperature on the Madden-Julian oscillation. J. Clim., 27, 8422-8443 doi: 10.1175/JCLI-D-14-00141.1 
Seo, H. and S.-P. Xie, 2011. Response and impact of equatorial ocean dynamics and tropical instability waves in the tropical Atlantic under global warming: A regional coupled downscaling study. J. Geophys. Res.: Oceans, 116, C03026. doi: 10.1029/2010JC006670

Seo, H. and S.-P. Xie. 2013. Impact of ocean warm layer thickness on the intensity of Hurricane Katrina in a regional coupled model. Meteorol. Atmos. Phys., 122, 19-32. doi: 10.1007/s00703013-0275-3

Seo, H., S.-P. Xie, R. Murtugudde, M. Jochum, and A. J. Miller. 2009. Seasonal effects of Indian Ocean freshwater forcing in a regional coupled model. J. Clim., 22, 6577-6596. doi: 10.1175/2009JCLI2990.1

Shinoda, T. 2005. Impact of diurnal cycle of solar radiation on intraseasonal SST variability in the western equatorial Pacific. J. Clim., 18, 2628-2636. doi: 10.1175/JCLI3432.1

Shinoda, T., T. Jensen, M. Flatau, and S. Chen. 2013. Surface wind and upper ocean variability associated with the Madden-Julian oscillation simulated by the coupled ocean/atmosphere mesoscale prediction system (COAMPS). Mon. Weather Rev., 141, 2290-2307. doi: 10.1175/MWR-D-1200273.1

Small, R. J., T. Campbell, J. Teixeira, T. A. Smith, J. Dykes, S. Chen, and R. Allard, 2011. Air-sea interaction in the Ligurian Sea: Assessment of a coupled ocean-atmosphere model using in situ data from LASIE07. Mon. Weather Rev., 139, 1785-1808. doi: 10.1175/2010MWR3431.1

Small, R. J., K. J. Richards, S.-P. Xie, P. Dutrieux, and T. Miyama. 2009. Damping of tropical instability waves caused by the action of surface currents on stress. J. Geophys. Res., 114, C04009. doi: 10.1029/2008JC005147

Small, R. J., S. P. de Szoeke, S. P. Xie, L. O’Neill, H. Seo, Q. Song, P. Cornillon et al. 2008. Air-sea interaction over ocean fronts and eddies. Dyn. Atmos. Oceans, 45, 274-319. doi: 10.1016/j.dynatmoce.2008.01.001

Smith, T. A., S. Chen, T. Campbell, P. Martin, W. E. Rogers, S. Gaberšek, D. Wang et al. 2013. Oceanwave coupled modeling in COAMPS-TC: A study of Hurricane Ivan (2004). Ocean Model., 69, 181-194. doi: 10.1016/j.ocemod.2013.06.003

Smith, R. B. and V. Grubišic. 1993. Aerial observations of Hawaii's wake. J. Atmos. Sci., 50(22), 3728-3750. doi: 10.1175/1520-0469(1993)050<3728:AOOHW>2.0.CO;2

Smith, G. C., F. Roy, and B. Brasnett. 2013. Evaluation of an operational ice-ocean analysis and forecasting system for the Gulf of St Lawrence. Q. J. R. Meteorol. Soc., 139(671), 419-433. doi: 10.1002/qj.1982

Stern, M. 1965. Interaction of a uniform wind stress with a geostrophic vortex. Deep-Sea Res. Oceanogr. Abstr., 12, 355-367. doi: 10.1016/0011-7471(65)90007-0

Subramanian, A. C., M. Jochum, A. J. Miller, R. Murtugudde, R. B. Neale, and D. E. Waliser. 2011. The Madden Julian oscillation in CCSM4. J. Clim., 24, 6261-6282. doi: 10.1175/JCLI-D-11-00031.1

SWAN Team. 2010. SWAN Scientific and Technical Documentation, SWAN Cycle III Version 40.81. Delft, the Netherlands: Delft University of Technology, 118 pp. Available: http://www. swan.tudelft.nl

Sweet, W., R. Fett, J. Kerling, and P. La Violette. 1981. Air-sea interaction effects in the lower troposphere across the north wall of the Gulf Stream. Mon. Weather Rev., 109, 1042-1052. doi: 10.1175/1520-0493(1981)109<1042:ASIEIT>2.0.CO;2

Taylor, P. K. and M. J. Yelland. 2001. The dependence of sea surface roughness on the height and steepness of the waves. J. Phys. Oceanogr., 31(2), 572-590. doi: 10.1175/15200485(2001)031<0572:TDOSSR > 2.0.CO;2 
Teague, W. J., E. Larosz, D. W. Wang, and D. A. Mitchell. 2007. Observed oceanic response over the upper continental slope and outer shelf during Hurricane Ivan. J. Phys. Oceanogr., 37, 2181-2206. doi: $10.1175 / \mathrm{JPO} 3115.1$

Theurich, G., C. DeLuca, T. Campbell, F. Liu, K. Saint, M. Vertenstein, J. Chen et al. 2016. The Earth System Prediction Suite: Toward a coordinated U.S. modeling capability. Bull. Amer. Meteorol. Soc., 97(7), 1229-1247. doi: 10.1175/BAMS-D-14-00164.1

Tolman, H. L. 2009. User Manual and System Documentation of WAVEWATCH IIITM, Version 3.14. Technical Note, NOAA/NWS/NCEP/MMAB. Camp Springs, MD: U.S. Dept. of Commerce, $220 \mathrm{pp}$.

Veeramony, J., M. D. Orzech, K. L. Edwards, M. Gilligan, J. Choi, E. Terrill, and T. De Paolo. 2014. Navy nearshore ocean prediction systems. Oceanography, 27(3), 80-91. doi: 10.5670/oceanog.2014.70

Vialard, J. and P. Delecluse. 1998. An OGCM study for the TOGA decade. Part II: Barrier-layer formation and variability. J. Phys. Oceanogr., 28, 1089-1106. doi: 10.1175/1520-0485(1998) 028<1089:AOSFTT $>2.0 . \mathrm{CO} ; 2$

Vianna, M. L., V. V. Menezes, A. B. Pezza, and I. Simmonds. 2010. Interactions between Hurricane Catarina (2004) and warm core rings in the South Atlantic Ocean. J. Geophys. Res., 115, C07002. doi: 10.1029/2009JC005974

Vitart, F. and F. Molteni. 2010. Simulation of the Madden-Julian oscillation and its teleconnections in the ECMWF forecast system. Q. J. R. Meteorol. Soc., 136, 842-855.

Waliser, D. E., R. Murtugudde, and L. E. Lucas. 2003. Indo-Pacific Ocean response to atmospheric intraseasonal variability: 1. Austral summer and the Madden-Julian Oscillation. J. Geophys. Res., 108, 3160. doi: 10.1029/2002JC001620

Waliser, D. E., K. Lau, and J. H. Kim. 1999. The influence of coupled sea surface temperatures on the Madden-Julian oscillation: A model perturbation experiment. J. Atmos. Sci., 56, 333-358. doi: 10.1175/1520-0469(1999)056<0333:TIOCSS > 2.0.CO;2

Wallace, J. M., T. P. Mitchell, and C. Deser. 1989. The influence of sea surface temperature on surface wind in the eastern equatorial Pacific: Seasonal and interannual variability. J. Clim., 2, 1492-1499. doi: 10.1175/1520-0442(1989)002<1492:TIOSST>2.0.CO;2

Warner, J. C., B. Armstrong, R. He, and J. B. Zambon. 2010. Development of a Coupled OceanAtmosphere-Wave-Sediment Transport (COAWST) modeling system. Ocean Model., 35(3), 230244. doi: 10.1016/j.ocemod.2010.07.010

Warner, J. C., C. R. Sherwood, H. G. Arango, and R. P. Signell. 2005. Performance of four turbulence closure models implemented using a generic length scale method. Ocean Model., 8(1), 81-113. doi: 10.1016/j.ocemod.2003.12.003

Weller, R. A., J. T. Farrar, J. Buckley, S. Mathew, R. Venkatesan, J. Sree Lekha, D. Chaudhuriet al. 2016. Air-sea interaction in the Bay of Bengal. Oceanography, 29(2), 28-37. doi: 10.5670/ oceanog. 2016.36

Woolnough, S. J., F. Vitart, and M. Balmaseda. 2007. The role of the ocean in the Madden-Julian Oscillation: Implications for MJO prediction. Q. J. R. Meteorol. Soc., 133, 117-128. doi: 10.1002/qj.4 Xie, S.-P. 2004. Satellite observations of cool ocean-atmosphere interaction. Bull. Amer. Meteor. Soc., 85, 195-209. doi: 10.1175/BAMS-85-2-195

Xie, S.-P., T. Miyama, Y. Wang, H. Xu, S. P. de Szoeke, R. J. Small, K. J. Richards et al. 2007. A regional ocean-atmosphere model for eastern Pacific climate: Towards reducing tropical biases. J. Clim., 20, 1504-1522. doi: 10.1175/JCLI4080.1 
Yu, Z. and J. P. McCreary. 2004. Assessing precipitation products in the Indian Ocean using an ocean model. J. Geophys. Res.: Oceans, 109, C05013. doi: 10.1029/2003JC002106

Yu, Z., J. P. McCreary, Jr., and J. A. Proehl. 1995. Meridional asymmetry and energetics of tropical instability waves. J. Phys. Oceanogr., 25, 2997-300 doi: 10.1175/1520-0485(1995)025<2997: MAAEOT $>2.0 . \mathrm{CO} ; 2$

Zhang, C., 2005. Madden-Julian oscillation. Rev. Geophys., 43, RG2003. doi: 10.1029/ 2004RG000158

Zhang, C., M. Dong, S. Gualdi, H. H. Hendon, E. D. Maloney, A. Marshall, K. R. Sperber, and W. Wang. 2006. Simulations of the Madden-Julian oscillation by four pairs of coupled and uncoupled global models. Clim. Dyn., 27, 573-592. doi: 10.1007/s00382-006-0148-2

Received: 5 October 2015; revised: 20 June 2017.

Editor's note: Contributions to The Sea: The Science of Ocean Prediction are being published separately in special issues of Journal of Marine Research and will be made available in a forthcoming supplement as Volume 17 of the series. 